\title{
Convergence of the invariant scheme of the method of fundamental solutions for two-dimensional potential problems in a Jordan region
}

\author{
Hidenori Ogata • Masashi Katsurada
}

Received: 19 September 2012 / Revised: 9 September 2013 / Published online: 24 September 2013 C The Author(s) 2013. This article is published with open access at Springerlink.com

\begin{abstract}
We examine the invariant scheme of the method of fundamental solutions for two-dimensional potential problems, that is, Dirichlet boundary value problems of the Laplace equation in a Jordan region, with the charge points and the collocation points obtained by a conformal mapping of the exterior of a disk to the exterior of the problem region. By a theoretical error analysis, we show that the approximate solution of the invariant scheme converges to the exact solution exponentially and some unnatural assumptions needed in the conventional scheme are removed in the convergence theorem of the invariant scheme.
\end{abstract}

Keywords Method of fundamental solutions - Charge simulation method - Laplace equation · Invariant scheme $\cdot$ Conformal mapping

Mathematics Subject Classification (2000) 65 N80 - 65N35 - 65N12 - 35J05 . 35J08

This work was supported by JSPS KAKENHI Grant Number 22540116.

H. Ogata $(\varangle)$

The Department of Communication Engineering and Informatics, Graduate School of Informatics and Engineering, The University of Electro-Communications, 1-5-1 Chofu-ga-Oka,

Chofu 182-8585, Japan

e-mail: ogata@im.uec.ac.jp

M. Katsurada

The Department of Mathematics, School of Science and Technology,

Meiji University, 1-1-1 Higashi-Mita, Kawasaki 214-8571, Japan 


\section{Introduction}

In this paper, we consider the "invariant scheme" of the method of fundamental solutions (MFS) for two-dimensional potential problems, that is, Dirichlet boundary value problems of the Laplace equation in a Jordan region, and we propose an arrangement of the points used in the method by a conformal mapping. We consider the two-dimensional Dirichlet problem of the Laplace equation

$$
\begin{cases}\triangle u=0 & \text { in } \Omega \\ u=f & \text { on } \Gamma\end{cases}
$$

where $\Omega$ is a region in the two-dimensional Euclidean plane and the boundary $\Gamma=\partial \Omega$ is a closed Jordan curve. Throughout in this paper, we equalize the two-dimensional Euclidean space $\mathbb{R}^{2}$ to the complex plane $\mathbb{C}^{1}$. The MFS gives an approximate solution of the potential problems (1) by a linear combination of the two-dimensional fundamental solutions of the Laplace operator

$$
u(z) \simeq u_{N}^{(\mathrm{C})}(z)=-\frac{1}{2 \pi} \sum_{j=1}^{N} Q_{j} \log \left|z-\zeta_{j}\right|,
$$

where $\zeta_{j} \in \mathbb{C} \backslash \bar{\Omega}(j=1,2, \ldots, N)$ are called the "charge points" and given by the user and $Q_{j} \in \mathbb{R}(j=1,2, \ldots, N)$ are called the "charges" determined by the way given below. We call the approximation (2) the "conventional scheme" of the MFS. We remark that the approximate solution $u_{N}$ satisfies the Laplace equation $\Delta u_{N}^{(\mathrm{C})}=0$ exactly in the problem region $\Omega$. Regarding the Dirichlet boundary condition, we choose the charges $Q_{j}$ by the collocation method so that $u_{N}^{(\mathrm{C})}$ satisfies the boundary condition approximately. Namely, we choose boundary points $z_{i} \in \Gamma(i=1,2, \ldots, N)$ which are called the "collocation points" and determine the charges $Q_{j}$ by the collocation equation

$$
u_{N}^{(\mathrm{C})}\left(z_{i}\right)=f\left(z_{i}\right) \quad(i=1,2, \ldots, N),
$$

which is equivalent to the system of linear equations for $Q_{j}$

$$
\begin{aligned}
& {\left[\begin{array}{llll}
G_{11} & G_{12} & \cdots & G_{1 N} \\
G_{21} & G_{22} & \cdots & G_{2 N} \\
\vdots & \vdots & & \vdots \\
G_{N 1} & G_{N 2} & \cdots & G_{N N}
\end{array}\right]\left[\begin{array}{l}
Q_{1} \\
Q_{2} \\
\vdots \\
Q_{N}
\end{array}\right]=\left[\begin{array}{l}
f\left(z_{1}\right) \\
f\left(z_{2}\right) \\
\vdots \\
f\left(z_{N}\right)
\end{array}\right],} \\
& G_{i j}=-\frac{1}{2 \pi} \log \left|z_{i}-\zeta_{j}\right|
\end{aligned}
$$

\footnotetext{
1 Throughout this paper, we denote the set of all the positive integers by $\mathbb{N}$, the set of all the integers by $\mathbb{Z}$, the set of all the real numbers by $\mathbb{R}$ and the set of all the complex numbers by $\mathbb{C}$.
} 
As shown above, the MFS is a very simple method and its computational cost is low. In addition, as shown in [5], this method achieves high accuracy such as exponential convergence under some conditions. Due to these advantages, the MFS is used widely in science and engineering.

However, the conventional scheme of the MFS lacks the very basic natural property that the solution is invariant with respect to trivial affine transformation such as scaling of coordinates

$$
z \rightarrow \alpha z \text { and } \zeta_{j} \rightarrow \alpha \zeta_{j}
$$

where $\alpha(\neq 0)$ is a real constant, and the origin shift for the boundary data

$$
f(z) \rightarrow f(z)+c
$$

where $c$ is a constant. The exact solution $u$ of the potential problem (1) transforms

$$
u(z) \rightarrow u(\alpha z)=u(z)
$$

under the transformation (5) and

$$
u(z) \rightarrow u(z)+c
$$

under the transformation (6), which, however, is not the case with the approximate solution $u_{N}^{(\mathrm{C})}$ of the conventional scheme (2) of the MFS. Murota gave a solution to this problem, that is, a scheme of the MFS which is invariant under the trivial affine transformations (5) and (6) [6,7]. The scheme, which is called the "invariant scheme", gives an approximate solution of the problem (1) in the form

$$
u(z) \simeq u_{N}^{(\mathrm{I})}(z)=C_{0}-\frac{1}{2 \pi} \sum_{j=1}^{N} Q_{j} \log \left|z-\zeta_{j}\right|
$$

where $\zeta_{j} \in \mathbb{C} \backslash \bar{\Omega}(j=1,2, \ldots, N)$ are the charge points given by the user, $C_{0} \in \mathbb{R}$ is a constant and $Q_{j} \in \mathbb{R}(j=1,2, \ldots, N)$ are the charges subject to the constraint

$$
\sum_{j=1}^{N} Q_{j}=0
$$

The approximate solution $u_{N}^{(\mathrm{I})}$ of the invariant scheme (7) also satisfies the Laplace equation exactly in the problem region $\Omega$ and, regarding the boundary condition, we choose the constants $C_{0}$ and $Q_{j}(j=1,2, \ldots, N)$ by the collocation method so that $u_{N}$ satisfies the boundary condition approximately. Namely, we choose the "collocation points" $z_{i} \in \Gamma(i=1,2, \ldots, N)$ and we determine the constants $C_{0}$ and $Q_{j}(j=1,2, \ldots, N)$ by the collocation equation 


$$
u_{N}^{(\mathrm{I})}\left(z_{i}\right)=f\left(z_{i}\right) \quad(i=1,2, \ldots, N),
$$

and the Eq. (8), which are equivalent to the system of linear equations for $C_{0}$ and $Q_{j}$

$$
\begin{aligned}
& {\left[\begin{array}{lllll}
G_{11} & G_{12} & \cdots & G_{1 N} & 1 \\
G_{21} & G_{22} & \cdots & G_{2 N} & 1 \\
\vdots & \vdots & & \vdots & \vdots \\
G_{N 1} & G_{N 2} & \cdots & G_{N N} & 1 \\
1 & 1 & \cdots & 1 & 0
\end{array}\right]\left[\begin{array}{l}
Q_{1} \\
Q_{2} \\
\vdots \\
Q_{N} \\
C_{0}
\end{array}\right]=\left[\begin{array}{l}
f\left(z_{1}\right) \\
f\left(z_{2}\right) \\
\vdots \\
f\left(z_{N}\right) \\
0
\end{array}\right],} \\
& G_{i j}=-\frac{1}{2 \pi} \log \left|z_{i}-\zeta_{j}\right|(i, j=1,2, \ldots, N) .
\end{aligned}
$$

The approximate solution of the invariant scheme $u_{N}^{(\mathrm{I})}$ satisfies the invariance properties of the exact solution of the potential problem. In fact, under the coordinate scaling (5), the approximate solution $u_{I}^{(\mathrm{I})}(z)$ transforms as

$$
\begin{aligned}
u_{N}^{(\mathrm{I})}(z) & =C_{0}-\frac{1}{2 \pi} \sum_{j=1}^{N} Q_{j} \log \left|z-\zeta_{j}\right| \\
& \rightarrow C_{0}-\frac{1}{2 \pi} \sum_{j=1}^{N} Q_{j} \log \left|\alpha\left(z-\zeta_{j}\right)\right| \\
& =u_{N}^{(\mathrm{I})}(z)-\frac{1}{2 \pi} \log |\alpha| \underbrace{\sum_{j=1}^{N} Q_{j}}_{0}=u_{N}^{(\mathrm{I})}(z)
\end{aligned}
$$

due to the constraint (8). And, under the origin shift (6), the approximate solution $u_{N}^{(\mathrm{I})}(z)$ transforms as $u_{N}^{(\mathrm{I})}(z) \rightarrow u_{N}^{(\mathrm{I})}(z)+c$ since the constants transforms as $C_{0} \rightarrow$ $C_{0}+c$ and $Q_{j} \rightarrow Q_{j}(j=1,2, \ldots, N)$.

It is important to choose the charge points $\zeta_{j}$ and the collocation points $z_{i}$ suitably for a good approximation in the both two schemes of the MFS. In the special case that the problem region $\Omega$ is a disk

$$
\Omega=D_{\rho}=\{z \in \mathbb{C}|| z \mid<\rho\} \quad(\rho>0),
$$

it is natural to choose the charge points $\zeta_{j}$ and the collocation points $z_{i}$ uniformly on concentric circles, that is,

$$
\zeta_{j}=\sigma \rho \omega^{j}, \quad z_{j}=\rho \omega^{j} \quad(j=1,2, \ldots, N),
$$

where $\omega=\mathrm{e}^{2 \pi \mathrm{i} / N}$ and $\sigma$ is a real constant called the "assignment parameter" such that $\sigma>1$. We call the positioning of the charge points and the collocation points an "equi-distant equally phased arrangement" of assignment parameter $\sigma$. It is known theoretically that the method of fundamental solutions works quite well if we choose 
the charge points $\zeta_{j}$ and the collocation points $z_{i}$ as (11) as in the theorems below. For the conventional scheme, we have the following theorem [5].

Theorem 1 We consider the conventional scheme of the MFS applied to a potential problem (1) in a disk region $D_{\rho}$. We assume that the charge points $\zeta_{j}$ and the collocation points $z_{i}$ of the MFS are in an equi-distant equally phased arrangement (11) of assignment parameter $\sigma>1$. Then, the following (i) and (ii) hold true.

(i) If we assume that $(\sigma \rho)^{N}-\rho^{N} \neq 1$, then the collocation equation (4) of the conventional scheme has a unique solution.

(ii) We further assume that $\sigma \rho \neq 1$ and the Fourier coefficients of the boundary data $f$

$$
\widehat{f}(n)=\int_{0}^{1} f\left(\rho \mathrm{e}^{2 \pi \mathrm{i} \tau}\right) \mathrm{e}^{-2 n \pi \mathrm{i} \tau} \mathrm{d} \tau \quad(n \in \mathbb{Z})
$$

decay exponentially, that is, there exist constants $A_{f}>0$ and $a(0<a<1)$ such that

$$
|\widehat{f}(n)| \leq A_{f} a^{|n|} \quad(\forall n \in \mathbb{Z})
$$

Then, the following error estimate of the conventional scheme of the MFS holds.

$$
\sup _{z \in D_{\rho}}\left|u(z)-u_{N}(z)\right| \leq A_{f} C_{C}(\rho, a, \sigma) \times \begin{cases}\sigma^{-N} & \text { if } \sigma<a^{-1 / 2} \\ N \sigma^{-N} & \text { if } \sigma=a^{-1 / 2} \\ a^{N / 2} & \text { if } \sigma>a^{-1 / 2}\end{cases}
$$

where $C_{\mathrm{C}}(\rho, a, \sigma)$ is a positive constant depending on $\rho$, a and $\sigma$ only.

For the invariant scheme, we have the following theorem ${ }^{2}$.

Theorem 2 We consider the invariant scheme of the MFS applied to a potential problem (1) in a disk region $D_{\rho}$. We assume that the charge points $\zeta_{j}$ and the collocation points $z_{i}$ of the MFS are in an equi-distant equally phased arrangement (11) of assignment parameter $\sigma>1$. Then, the following (i) and (ii) hold true.

(i) The collocation equation (4) of the invariant scheme with the Eq. (8) has a unique solution.

(ii) We further assume that the Fourier coefficients of the boundary data $f$ decay exponentially as in (12). Then, for the error of the invariant scheme, we have the inequality

$$
\sup _{z \in D_{\rho}}\left|u(z)-u_{N}^{(\mathrm{I})}(z)\right| \leq A_{f} C_{\mathrm{I}}(\rho, a, \sigma) \times \begin{cases}\sigma^{-N} & \text { if } \sigma<a^{-1 / 2} \\ N \sigma^{-N} & \text { if } \sigma=a^{-1 / 2} \\ a^{N / 2} & \text { if } \sigma>a^{-1 / 2}\end{cases}
$$

where $C_{\mathrm{I}}(\rho, a, \sigma)$ is a positive constant depending on $\rho$, a and $\sigma$ only.

2 The result on the convergence of the invariant scheme is not given in the previous papers as far as the authors know, but we can obtain the convergence theorem easily in a way similar to the proof of Theorem 1 . 
We remark that the convergence theorem of the invariant scheme for a disk region $D_{\rho}$ does not require the somewhat unnatural assumptions that

$$
(\sigma \rho)^{N}-\rho^{N} \neq 1 \text { and } \sigma \rho \neq 1
$$

which are needed in the convergence theorem of the conventional scheme for a disk region. It may be a reflection of the fact that the solution $u_{N}^{(\mathrm{I})}$ of the invariant scheme has invariance properties under trivial transformations (5) and (6).

In the cases of general regions, it is a difficult problem how to choose the charge points and the collocation points since it is not trivial how to position the points uniformly. Katsurada gave a solution of positioning the points [4]. Namely, he proposed to use the images of points in an equi-distant equally phased arrangement (11), that is, he proposed to use the points

$$
\zeta_{j}=\Psi\left(\sigma \rho \omega^{j}\right) \text { and } z_{j}=\Psi\left(\rho \omega^{j}\right) \quad(j=1,2, \ldots, N),
$$

with a conformal mapping

$$
\Psi:\{w \in \mathbb{C}|| w \mid>\rho\} \rightarrow \mathbb{C} \backslash \bar{\Omega},
$$

which is assured to exist by Riemann's mapping theorem and admits a conformal extension to $\{w \in \mathbb{C}|| w \mid \geq \alpha \rho\}(0<\alpha<1)$, as the charge points $\zeta_{j}$ and the collocation points $z_{j}$ of the conventional scheme of the MFS, and he presented a convergence theorem for the conventional scheme with the charge points and the collocation points (13). Katsurada also proposed to use the charge points and the collocation points of the MFS obtained by an interior conformal mapping [3].

The purpose of this paper is to extend Katsurada's result to the invariant scheme of the MFS. Namely, we propose to use the points (14) as the charge points $\zeta_{j}$ and the collocation points $z_{j}$ also in the invariant scheme of the MFS and give a convergence theorem of this scheme. The convergence theorem for the conventional scheme (Theorem 3.1 of [4]) requires the assumptions that

$$
\sigma \rho \neq 1 \text { and the capacity of the curve }\left\{\Psi\left(\sigma \rho \mathrm{e}^{2 \pi \mathrm{i} \tau}\right) \in \mathbb{C} \mid 0 \leq \tau \leq 1\right\} \neq 1 \text {, }
$$

which are rather unnatural from a physical point of view. It is, however, shown in this paper that the convergence theorem for the invariant scheme does not need these unnatural assumptions. It is an advantage of the invariant scheme of the MFS compared with the conventional scheme, and it may be a reflection of the fact that the invariant scheme has the invariance properties under the trivial affine transformations (5) and (6).

The contents of this paper are as follows. In Sect. 2, we prepare some notations for the analysis of the invariant scheme of the MFS. Especially, we introduce a Hilbert space to which the exact solution $u$, the approximate solution $u_{N}^{(\mathrm{I})}$ of the invariant scheme and the boundary data $f$ belong and whose norm is used to estimate the error of the invariant scheme. In Sect. 3, we present main theorems of this paper, which show the convergence of the invariant scheme of the MFS. We present the proof of the 
theorems in Sect. 4. In Sect. 5, we summarize this paper and give some concluding remarks.

\section{Preliminaries}

Throughout this paper, we use the notation $S^{1}=\mathbb{R} / \mathbb{Z}$. Functions defined on $S^{1}$ are periodic functions of period 1 . We reduce our problem to a problem of approximating functions on $S^{1}$ and use a technique based on the Fourier analysis for our purpose.

Following [4], we prepare a family of functions for the analysis of the invariant scheme of the MFS. Let $\mathscr{T}$ be the set of all the real valued finite Fourier series, that is,

$$
\mathscr{T}=\left\{\varphi(\tau)=\sum_{n \in \mathbb{Z}} \widehat{\varphi}(n) \mathrm{e}^{2 n \pi \mathrm{i} \tau} \mid \widehat{\varphi}(n) \in \mathbb{C}\right.
$$

are zeros except for a finite number of them and $\widehat{\varphi}(-n)=\overline{\widehat{\varphi}(n)} \quad(\forall n \in \mathbb{Z})\}$.

For each $(\epsilon, s) \in(0,+\infty) \times \mathbb{R}$, we define the norm $\|\cdot\|_{\epsilon, s}$ on $\mathscr{T}$ by

$$
\|\varphi\|_{\epsilon, s}=\left\{\sum_{n \in \mathbb{Z}}|\widehat{\varphi}(n)|^{2} \epsilon^{2|n|} \underline{n}^{2 s}\right\}^{1 / 2}
$$

where

$$
\underline{n}= \begin{cases}2 \pi|n| & (n \neq 0) \\ 1 & (n=0)\end{cases}
$$

and define the Hilbert space $\mathscr{X}_{\epsilon, s}$ as the completion of $\mathscr{T}$ in the norm $\|\cdot\|_{\epsilon, s}$. Hilbert space $\mathscr{X}_{\epsilon, s}$ is originally introduced by Arnold for the analysis of the splinetrigonometric-Galerkin method [1].

We also prepare the following notations.

1. We define the order relations " $\geq$ " and " $>$ " on $(0,+\infty) \times \mathbb{R}$ respectively by

$$
\left(\epsilon_{1}, s_{1}\right) \geq\left(\epsilon_{2}, s_{2}\right) \Longleftrightarrow \epsilon_{1}>\epsilon_{2} \text { or }\left(\epsilon_{1}=\epsilon_{2} \text { and } s_{1} \geq s_{2}\right)
$$

and

$$
\left(\epsilon_{1}, s_{1}\right)>\left(\epsilon_{2}, s_{2}\right) \Longleftrightarrow\left(\epsilon_{1}, s_{1}\right) \geq\left(\epsilon_{2}, s_{2}\right) \text { and }\left(\epsilon_{1}, s_{1}\right) \neq\left(\epsilon_{2}, s_{2}\right)
$$

that is, the order relations " $\geq$ " and " $>$ " are the lexicographic order relations.

2. We define the sets $\Lambda_{N}$ and $\Delta_{N}$ for $N \in \mathbb{N}$ respectively by

$$
\Lambda_{N}=\left\{n \in \mathbb{Z} \mid-\frac{N}{2} \leq n<\frac{N}{2}\right\} \quad \text { and } \quad \Delta_{N}=\left\{\frac{n}{N} \in S^{1} \mid n \in \Lambda_{N}\right\} .
$$

Related to the functional spaces $\mathscr{X}_{\epsilon, s}$, we have the following lemma. 
Lemma 1 If $\left(\epsilon_{1}, s_{1}\right)>\left(\epsilon_{2}, s_{2}\right)$, then the natural inclusion $\mathscr{X}_{\epsilon_{1}, s_{1}} \hookrightarrow \mathscr{X}_{\epsilon_{2}, s_{2}}$ exists and is compact.

Proof It is easy to prove that $\mathscr{X}_{\epsilon_{1}, s_{1}} \subset \mathscr{X}_{\epsilon_{2}, s_{2}}$ if $\left(\epsilon_{1}, s_{1}\right)>\left(\epsilon_{2}, s_{2}\right)$. In order to prove that this inclusion is compact, we remark that we can equalize $\varphi \in \mathscr{X}_{\epsilon_{1}, s_{1}}$ to a sequence $\left\{\widehat{\varphi}(n) \epsilon_{1}^{|n|} \underline{n}^{s_{1}}\right\}_{n \in \mathbb{Z}} \in l^{2}$. Then, the inclusion mapping $\mathscr{X}_{\epsilon_{1}, s_{1}} \hookrightarrow \mathscr{X}_{\epsilon_{2}, s_{2}}$ is equalised to the mapping of $\left\{\widehat{\varphi}(n) \epsilon_{1}^{|n|} \underline{n}^{s_{1}}\right\} \in l^{2}$ to

$$
\left\{\widehat{\varphi}(n) \epsilon_{2}^{|n|} \underline{n}^{s_{2}}\right\}_{n \in \mathbb{Z}}=\left\{\widehat{\varphi}(n) \epsilon_{1}^{|n|} \underline{n}^{s_{1}} \cdot\left(\epsilon_{2} / \epsilon_{1}\right)^{|n|} \underline{n}^{s_{2}-s_{1}}\right\}_{n \in \mathbb{Z}} \in l^{2}
$$

Since the sequence $\left\{\left(\epsilon_{2} / \epsilon_{1}\right)^{|n|} \underline{n}^{s_{2}-s_{1}}\right\}_{n \in \mathbb{Z}}$ decreases monotonously as $n \rightarrow \pm \infty$, this mapping is compact.

Related to the expression of the approximate solution of the invariant scheme (7) and (8), we define the integral operator $A$ for function $q$ on $S^{1}$ by

$$
A q(\tau)=\widehat{q}(0)-\frac{1}{2 \pi} \int_{S^{1}} \log \left|\Psi\left(\rho \mathrm{e}^{2 \pi \mathrm{i} \tau}\right)-\Psi\left(\sigma \rho \mathrm{e}^{2 \pi \mathrm{i} \theta}\right)\right|\{q(\theta)-\widehat{q}(0)\} \mathrm{d} \theta
$$

Using the integral operator $A$, the approximate solution of the invariant scheme on the boundary $\Gamma$ is written as

$$
u_{N}^{(\mathrm{I})}\left(\Psi\left(\rho \mathrm{e}^{2 \pi \mathrm{i} \tau}\right)\right)=A q_{N}(\tau) \quad\left(\tau \in S^{1}\right)
$$

with

$$
q_{N}(\tau)=C_{0}+\sum_{j \in \Lambda_{N}} Q_{j} \delta\left(\tau-\frac{j}{N}\right)
$$

where $C_{0}$ and $Q_{j}\left(j \in \Lambda_{N}\right)$ are real constants such that

$$
\sum_{j \in \Lambda_{N}} Q_{j}=0
$$

and $\delta(\cdot)$ is the Dirac delta function. The constants $C_{0}$ and $Q_{j}\left(j \in \Lambda_{N}\right)$ are determined by the collocation equation

$$
u_{N}^{(\mathrm{I})}\left(\Psi\left(\rho \omega^{i}\right)\right)=f\left(\Psi\left(\rho \omega^{i}\right)\right) \quad\left(i \in \Lambda_{N}\right)
$$

and the Eq. (18). We denote the set of functions $q_{N}$ of the form (17) whose real constants $C_{0}$ and $Q_{j}\left(j \in \Lambda_{N}\right)$ satisfy the condition (18) by $\mathscr{D}_{N}$. Related to $\mathscr{D}_{N}$, we have the following lemma, which can be proved easily. 
Lemma 2 1. For arbitrary $q_{N} \in \mathscr{D}_{N}$, we have

$$
\begin{aligned}
\widehat{q}_{N}(n)= & \widehat{q}_{N}(m) \text { if } n \equiv m \bmod N \text { and } n, m \neq 0 \quad \bmod N . \\
& \widehat{q}_{N}(n)=0 \text { if } n \equiv 0 \bmod N \text { and } n \neq 0 .
\end{aligned}
$$

2. $\mathscr{D}_{N} \subset \mathscr{X}_{\epsilon, s}$ if $(\epsilon, s)<(1,-1 / 2)$.

In this paper, we express the solution of the potential problem (1) as a single layer potential

$$
u(z)=\widehat{q}(0)-\frac{1}{2 \pi} \int_{S^{1}} \log \left|z-\Psi\left(\sigma \rho \mathrm{e}^{2 \pi \mathrm{i} \theta}\right)\right|\{q(\theta)-\widehat{q}(0)\} \mathrm{d} \theta
$$

with charge density $q(\theta)\left(\theta \in S^{1}\right)$, and, then, we have

$$
u\left(\Psi\left(\rho \mathrm{e}^{2 \pi \mathrm{i} \tau}\right)\right)=f\left(\Psi\left(\rho \mathrm{e}^{2 \pi \mathrm{i} \tau}\right)\right)=A q(\tau) \quad\left(\tau \in S^{1}\right) .
$$

We regard the approximate solution $u_{N}^{(\mathrm{I})}$ by the invariant scheme as a single layer potential with discrete charge density $q_{N}(\simeq q)$ and estimate the error on the boundary $\left.\left(f-u_{N}^{(\mathrm{I})}\right)\right|_{\Gamma}$ by measuring the error of the charge $q_{N}-q$ by the norm $\|\cdot\|_{\epsilon^{\prime}, s^{\prime}}$ of a Hilbert space $\mathscr{X}_{\epsilon^{\prime}, s^{\prime}}$ for some $\left(\epsilon^{\prime}, s^{\prime}\right) \in(0,+\infty) \times \mathbb{R}$.

\section{Main theorem}

The following theorem is the main result of this paper.

Theorem 3 We consider the application of the invariant scheme of the MFS to the potential problem with the charge points $\zeta_{j}$ and the collocation points $z_{j}$ given by

$$
z_{j}=\Psi\left(\rho \omega^{j}\right), \quad \zeta_{j}=\Psi\left(\sigma \rho \omega^{j}\right) \quad\left(j \in \Lambda_{N}\right)
$$

where $\Psi$ is a conformal mapping

$$
\Psi:\{w \in \mathbb{C}|| w \mid>\rho\} \rightarrow \mathbb{C} \backslash \bar{\Omega},
$$

$\omega=\mathrm{e}^{2 \pi \mathrm{i} / N}$ and $\sigma$ is a constant such that $\sigma>1$. We assume the following conditions.

1. The conformal mapping $\Psi$ admits a conformal extension to $\{w \in \mathbb{C}|| w \mid \geq \alpha \rho\}$, where $\alpha$ is a constant such that $0<\alpha<1$.

1. $\sigma \alpha<1$.

Then, the following (i) and (ii) hold true.

(i) The collocation equation (19) of the invariant scheme with the Eq. (18) have a unique solution, that is, the coefficient matrix of the system of linear equations (10) with $\zeta_{j}$ and $z_{i}$ given by (22) is regular. 
(ii) We further assume that the real constants a and t satisfy

$$
1<a^{-1}<\sigma^{2}, \quad\left(a^{-1}, t\right)<\left(\alpha^{-1},-1 / 2\right)
$$

Then, there exists a constant $C>0$ such that, for sufficiently large $N \in \mathbb{N}$ and for arbitrary $f$ with $f_{\rho} \in \mathscr{X}_{a^{-1}, t}$, where $f_{\rho}$ is defined by $f_{\rho}(\tau)=f\left(\Psi\left(\rho \mathrm{e}^{2 \pi \mathrm{i} \tau}\right)\right)$, the following error estimate of the invariant scheme holds.

$$
\left\|f-u_{N}^{(\mathrm{I})}\right\|_{H^{s}(\Gamma)} \leq C\left\|f_{\rho}\right\|_{a^{-1}, t} N^{s-t} a^{N / 2},
$$

where $s$ is an arbitrary real constant, $H^{s}(\Gamma)$ is the set of functions $v$ defined on $\Gamma$ such that the function $v_{\rho}(\tau)=v\left(\Psi\left(\rho \mathrm{e}^{2 \pi \mathrm{i} \tau}\right)\right)$ defined on $S^{1}$ belongs to the usual Sobolev space $H^{s}\left(S^{1}\right)$ and $\|v\|_{H^{s}(\Gamma)}=\left\|v_{\rho}\right\|_{H^{s}\left(S^{1}\right)}\left(v \in H^{s}(\Gamma)\right)$.

It is interesting that the order of the convergence in (24) is, neglecting $N$ 's power, the same as the order of the convergence of the conventional scheme with $\sigma>a^{-1 / 2}$ and the invariant scheme with $\sigma>a^{-1 / 2}$ applied to problems in disk regions as shown respectively in Theorems 1 and 2 . We also remark that the condition that $f_{\rho} \in \mathscr{X}_{a^{-1}, t}$ is satisfied if and only if the boundary data $f(z)$ is an analytic function in the annulus $a \rho<|z|<\rho / a$.

Remark 1 From the viewpoint of actual computations, it is important to measure the error by the supremum norm. If $s>1 / 2$ and $v \in H^{s}(\Gamma)$, we have $v \in C(\Gamma)$ and $^{3}$

$$
\|v\|_{\infty} \leq C\|v\|_{H^{s}(\Gamma)} \quad\left(\forall v \in H^{S}(\Gamma)\right) .
$$

In fact, we have for $\theta \in S^{1}$

$$
\begin{aligned}
\left|v\left(\Psi\left(\rho \mathrm{e}^{2 \pi \mathrm{i} \theta}\right)\right)\right| & =\left|\sum_{n \in \mathbb{Z}} \widehat{v_{\rho}}(n) \mathrm{e}^{2 \pi \mathrm{i} \theta}\right| \leq \sum_{n \in \mathbb{Z}}\left|\widehat{v_{\rho}}(n)\right| \\
& =\sum_{n \in \mathbb{Z}} \underline{n}^{-s} \cdot\left|\widehat{v_{\rho}}(n)\right| \underline{n}^{s} \leq\left\{\sum_{n \in \mathbb{Z}} \underline{n}^{-2 s}\right\}^{1 / 2}\left\{\sum_{n \in \mathbb{Z}}\left|\widehat{v_{\rho}}(n)\right|^{2} \underline{n}^{2 s}\right\}^{1 / 2}
\end{aligned}
$$

and $\sum_{n \in \mathbb{Z}} \underline{n}^{-2 s}<\infty$ since $s>1 / 2$. Therefore, if $s>1 / 2$ and $f_{\rho} \in \mathscr{X}_{a^{-1}, t}$ we have

$$
\left\|f_{\rho}-\left(u_{N}^{(\mathrm{I})}\right)_{\rho}\right\|_{\infty} \leq C\left\|f_{\rho}\right\|_{a^{-1}, t} N^{s-t} a^{N / 2},
$$

and, by the principle of the maximum, we have

$$
\sup _{z \in \Omega}\left|u(z)-u_{N}^{(\mathrm{I})}(z)\right| \leq C\left\|f_{\rho}\right\|_{a^{-1}, t} N^{s-t} a^{N / 2} .
$$

\footnotetext{
3 Throughout this paper, the symbols " $C, C^{\prime}, \ldots$ " etc. denote positive constants and take different values in different lines.
} 
Remark 2 The condition that $f_{\rho} \in \mathscr{X}_{a^{-1}, t}$ is satisfied if and only if the boundary data $f(z)$ is an analytic function in a neighborhood of the boundary $\Gamma$. In fact, if $f(z)$ is analytic in the region $\left\{\Psi(w)\left|r_{0} \leq\right| w \mid \leq \rho^{2} / r_{0}\right\}$ with $r_{0}$ such that $\sigma^{-2} \rho<r_{0}<\rho$, we have

$$
\left|\widehat{f}_{\rho}(n)\right| \leq\left(\frac{r_{0}}{\rho}\right)^{|n|} \sup \left\{|f(\Psi(w))|\left|r_{0} \leq\right| w \mid \leq \frac{\rho^{2}}{r_{0}}\right\}
$$

and the condition that $f_{\rho} \in \mathscr{X}_{a^{-1}, t}$ holds for arbitrary $a$ such that $r_{0} / \rho<a<1$. Conversely, if the condition that $f_{\rho} \in \mathscr{X}_{a^{-1}, t}$ holds, let

$$
\mathscr{F}_{+}(w)=\sum_{n=0}^{\infty} \widehat{f}_{\rho}(n) w^{n}, \quad \mathscr{F}_{-}(w)=\sum_{n=1}^{\infty} \widehat{f}_{\rho}(-n) w^{-n}
$$

The function $\mathscr{F}_{+}(w)$ is analytic in a neighborhood of $\{w \in \mathbb{C}|| w \mid \leq 1\}$ and the function $\mathscr{F}_{-}(w)$ is analytic in a neighborhood of $\{w \in \mathbb{C}|| w \mid \geq 1\}$. Hence, the function $\mathscr{F}=\mathscr{F}_{+}+\mathscr{F}_{-}$is analytic in a neighborhood of the unit circle $\{w \in \mathbb{C}|| w \mid=1\}$. Let

$$
F(w)=\mathscr{F}\left(\frac{w}{\rho}\right)
$$

Then, the function $F(w)$ is analytic in a neighborhood of the circle $\{w \in \mathbb{C}|| w \mid=\rho\}$ and coincides with the transformed boundary data $f(\Psi(w))$ on the circle $\{w \in \mathbb{C}|| w \mid$ $=\rho\}$. It implies that the transformed boundary data $f(\Psi(w))$ is analytic in a neighborhood of the circle $\{w \in \mathbb{C}|| w \mid=\rho\}$ and, then, the boundary data $f(z)$ is analytic in a neighborhood of $\Gamma$.

Theorem 3 is obtained as a special case of the following theorem.

Theorem 4 Let $(\epsilon, s),(\delta, t) \in(0,+\infty) \times \mathbb{R}$, and $\sigma$ be the parameter appearing in (22) such that $\sigma>1$. We assume the following conditions.

1. The conformal mapping $\Psi$ of (23) admits a conformal extension to $\{w \in \mathbb{C}|| w \mid \geq$ $\alpha \rho\}(0<\alpha<1)$.

2. $\left(1, \frac{1}{2}\right)<(\delta, t)<\left(\frac{1}{\alpha},-\frac{1}{2}\right)$.

3. $\max \left\{\frac{\delta}{\sigma^{2}}, \frac{1}{\delta}\right\} \leq \epsilon \leq \min \left\{\delta, \frac{\sigma^{2}}{\delta}\right\}$. If $\epsilon=\delta$, we further assume $s<t$ and, if $\epsilon=\delta=\sigma$, we further assume $s<\frac{1}{2}$.

4. $\sigma \alpha<1$.

5. $\left(\alpha, \frac{3}{2}\right)<(\epsilon, s)$.

Then, the following (i) and (ii) hold true. 
(i) For arbitrary $\tilde{f} \in \mathscr{X}_{\delta, t}$ and sufficiently large $N \in \mathbb{N}$, there exists a unique function $q_{N} \in \mathscr{D}_{N}$ such that

$$
A q_{N}=\tilde{f} \text { on } \Delta_{N}
$$

(ii) There exists a constant $C>0$ such that

$$
\left\|\widetilde{f}-A q_{N}\right\|_{\epsilon, s} \leq C\|\widetilde{f}\|_{\delta, t} N^{P(\epsilon, s, \delta, t)}\left(\frac{\epsilon}{\delta}\right)^{N / 2}
$$

where $P(\epsilon, s, \delta, t)$ is defined by

$$
P(\epsilon, s, \delta, t)= \begin{cases}\max \{s-t,-t\} & \text { if } \delta \epsilon=1 \text { and } \sigma^{-1} \leq \delta<\sigma \\ \max \{s-t, s-1\} & \text { if } \delta \epsilon=\sigma^{2} \text { and } \sigma \leq \delta<\sigma^{2} \\ \max \{s-t,-1\} & \text { if } \epsilon=\sigma^{-2} \delta \text { and } \sigma<\delta<\sigma^{2} \\ \max \{s-t,-t,-1\} & \text { if }(\delta, \epsilon)=\left(\sigma, \sigma^{-1}\right) \\ \max \{s-t, s-1,-1\} & \text { if }(\delta, \epsilon)=\left(\sigma^{2}, 1\right) \\ s-t & \text { otherwise. }\end{cases}
$$

Remark 3 Since the definition of $P(\epsilon, s, \delta, t)$ by (27) is rather complicated, we rewrite (27) by using a figure. Figure 1 shows the region of $(\delta, \epsilon)$ satisfying the assumption of Theorem 4 and, using this figure, we can rewrite (27) as

$$
P(\epsilon, s, \delta, t)= \begin{cases}\max \{s-t,-t\} & \text { if }(\delta, \epsilon) \in H_{1} \backslash\left\{\left(\sigma, \sigma^{-1}\right)\right\} \\ \max \{s-t, s-1\} & \text { if }(\delta, \epsilon) \in H_{2} \backslash\left\{\left(\sigma^{2}, 1\right)\right\} \\ \max \{s-t,-1\} & \text { if }(\delta, \epsilon) \in L_{1} \backslash\left\{\left(\sigma, \sigma^{-1}\right),\left(\sigma^{2}, 1\right)\right\} \\ \max \{s-t,-t,-1\} & \text { if }(\delta, \epsilon)=\left(\sigma, \sigma^{-1}\right) \\ \max \{s-t, s-1,-1\} & \text { if }(\delta, \epsilon)=\left(\sigma^{2}, 1\right) \\ s-t & \text { otherwise. }\end{cases}
$$

Fig. 1 The region where $(\delta, \epsilon)$ exists (the shadowed area)

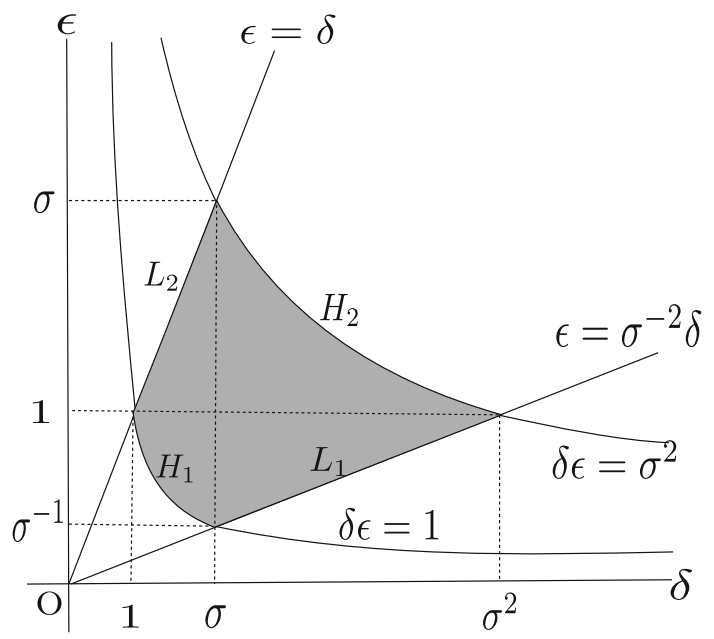


The aim of this paper is to prove Theorem 4 . Theorem 3 is obtained by putting $\delta=a^{-1}, \epsilon=1, u_{N}^{(\mathrm{I})}\left(\Psi\left(\rho \mathrm{e}^{2 \pi \mathrm{i} \tau}\right)\right)=A q_{N}(\tau)$ and $\widetilde{f}=f_{\rho}$ in Theorem 4, whose assumptions are satisfied as follows.

1. The first assumption is satisfied.

2. The relation $(\delta, t)=\left(a^{-1}, t\right)<\left(\alpha^{-1},-1 / 2\right)$ holds true and the relation $(\delta, t)=$ $\left(a^{-1}, t\right)>(1,1 / 2)$ holds true for arbitrary $t \in \mathbb{R}$ since $1<a^{-1}\left(<\sigma^{2}\right)$.

3. The relation $\epsilon>\max \left\{\sigma^{-2} \delta, \delta^{-1}\right\}$ holds true since $\sigma^{-2} \delta=\sigma^{-2} a^{-1}<1=\epsilon$ and $\delta^{-1}=a<1=\epsilon$, which are obtained by $1<a^{-1}<\sigma^{2}$. The relation $\epsilon<\min \left\{\delta, \sigma^{2} \delta^{-1}\right\}$ holds true since $\delta=a^{-1}>1=\epsilon$ and $\sigma^{2} \delta^{-1}=\sigma^{2} a>$ $1=\epsilon$, which is obtained by $1<a^{-1}<\sigma^{2}$. In addition, from the inequality $\max \left\{\sigma^{-2} \delta, \delta^{-1}\right\}<\epsilon<\min \left\{\delta, \sigma^{2} \delta^{-1}\right\}$, the definition of $P(\epsilon, s, \delta, t)(28)$ and Fig. 1, we find $P(\epsilon, s, \delta, t)=P\left(1, s, a^{-1}, t\right)=s-t$.

4. The fourth assumption is satisfied.

5. Since $\epsilon=1>\alpha$, we have $(\epsilon, s)>(\alpha, 3 / 2)$ for arbitrary $s \in \mathbb{R}$.

We remark that the right hand side of (26) decays as $N$ increases, that is,

$$
N^{P(\epsilon, s, \delta, t)}\left(\frac{\epsilon}{\delta}\right)^{N / 2}=\mathrm{o}(1) \quad \text { as } N \rightarrow \infty
$$

In fact, we have $\epsilon / \delta \leq 1$ and, if $\epsilon=\delta$, we have

$$
P(\epsilon, s, \delta, t)= \begin{cases}\max \{s-t,-t\} & \text { if }(\delta, \epsilon)=(1,1) \\ \max \{s-t, s-1\} & \text { if }(\delta, \epsilon)=(\sigma, \sigma) \\ s-t & \text { otherwise. }\end{cases}
$$

Then, we have $P(\epsilon, s, \delta, t)<0$ from the assumptions of the theorem.

Remark 4 In the error analysis of the conventional scheme in [4], the solution $u$ is represented as a single layer potential

$$
u(z)=-\frac{1}{2 \pi} \int_{S^{1}} \log \left|z-\Psi\left(\sigma \rho \mathrm{e}^{2 \pi \mathrm{i} \theta}\right)\right| q(\theta) \mathrm{d} \theta
$$

and the integral operator $A_{\mathrm{C}}$ given by

$$
A_{\mathrm{C}} q(\tau)=-\frac{1}{2 \pi} \int_{S^{1}} \log \left|\Psi\left(\rho \mathrm{e}^{2 \pi \mathrm{i} \tau}\right)-\Psi\left(\sigma \rho \mathrm{e}^{2 \pi \mathrm{i} \theta}\right)\right| q(\theta) \mathrm{d} \theta
$$

is used for the error estimation. Theorem 3.2 of [4] presents the unique solvability of the collocation equation and the convergence of the same order as (26) of the conventional scheme of the MFS under the assumptions of Theorem 3 and, in addition, the assumption that

$\sigma \rho \neq 1$ and the capacity of the curve $\left\{\Psi\left(\sigma \rho \mathrm{e}^{2 \pi \mathrm{i} \tau}\right) \mid \tau \in S^{1}\right\} \neq 1$ 
which is unnatural from physical point of view. We remark that, in Theorem 3, these unnatural assumptions are removed.

We now present the sketch of the proof. First, we consider the special case that the problem region is a disk $D_{\rho}=\{z \in \mathbb{C}|| z \mid<\rho\}$, that is, the conformal mapping $\Psi$ is the identity. In this case, the integral operator $A$ is the operator $L$ given by

$$
L q(\tau)=\widehat{q}(0)-\frac{1}{2 \pi} \int_{S^{1}} \log \left|\rho \mathrm{e}^{2 \pi \mathrm{i} \tau}-\sigma \rho \mathrm{e}^{2 \pi \mathrm{i} \theta}\right|\{q(\theta)-\widehat{q}(0)\} \mathrm{d} \theta
$$

and we can show the existence of a unique function $q_{N} \in \mathscr{D}_{N}$ such that $L q_{N}=$ $\widetilde{f}$ on $\Delta_{N}$, that is, the unique solvability of the collocation equation (9) with the Eq. (8) and estimate the error of the approximate solution $\left\|\widetilde{f}-L q_{N}\right\|_{\epsilon, s}$ by elementary calculus of the Fourier series. Second, in the case of a general region $\Omega$, we split the operator $A$ as $A=L+K$, where $K$ is the integral operator given by

$$
K q(\tau)=-\frac{1}{2 \pi} \int_{S^{1}} \log \left|\frac{\Psi\left(\rho \mathrm{e}^{2 \pi \mathrm{i} \tau}\right)-\Psi\left(\sigma \rho \mathrm{e}^{2 \pi \mathrm{i} \theta}\right)}{\rho \mathrm{e}^{2 \pi \mathrm{i} \tau}-\sigma \rho \mathrm{e}^{2 \pi \mathrm{i} \theta}}\right|\{q(\theta)-\widehat{q}(0)\} \mathrm{d} \theta .
$$

Then, regarding $K$ as a compact perturbation of the isomorphism $L$, we show that $A$ is an isomorphism, there exists a unique function $q_{N} \in \mathscr{D}_{N}$ such that $A q_{N}=\widetilde{f}$ on $\Delta_{N}$, that is, there exists a unique solution of the collocation equation (9) with the Eq. (8) and estimate the error $\left\|\widetilde{f}-A q_{N}\right\|_{\epsilon, s}$ by the application of the Riesz-Schauder theory.

\section{Proof of the main theorem}

\subsection{Step 1 - case of a disk region}

First, we consider the special case that the region $\Omega$ is a disk $D_{\rho}$ and the conformal mapping $\Psi$ is the identity mapping. In this case, the approximate solution of the invariant scheme is given by

$$
u_{N}^{(\mathrm{I})}(z)=C_{0}-\frac{1}{2 \pi} \sum_{j \in \Lambda_{N}} Q_{j} \log \left|z-\sigma \rho \omega^{j}\right|,
$$

where the constants $C_{0}, Q_{j}\left(j \in \Lambda_{N}\right)$ are determined by the collocation equation

$$
u_{N}^{(\mathrm{I})}\left(\rho \omega^{i}\right)=f\left(\rho \omega^{i}\right) \quad\left(i \in \Lambda_{N}\right)
$$

and the constraint

$$
\sum_{j \in \Lambda_{N}} Q_{j}=0
$$


The integral operator $A$ is the operator $L$ given by (29). Using the function

$$
G(\theta)=-\frac{1}{2 \pi} \log \left|\sigma \rho-\rho \mathrm{e}^{2 \pi \mathrm{i} \theta}\right|,
$$

the integral operator $L$ is given by the convolution

$$
L q=\widehat{q}(0)+G *\{q-\widehat{q}(0)\} .
$$

Since the Fourier coefficients of $G(\theta)$ are given by [5]

$$
\widehat{G}(n)= \begin{cases}\frac{\sigma^{-|n|}}{4 \pi|n|} & (n \neq 0) \\ -\frac{1}{2 \pi} \log (\sigma \rho) & (n=0),\end{cases}
$$

the Fourier coefficients of $L q$ are given by

$$
\widehat{L q}(n)= \begin{cases}\frac{\sigma^{-|n|}}{4 \pi|n|} \widehat{q}(n) & (n \neq 0) \\ \widehat{q}(0) & (n=0) .\end{cases}
$$

Therefore, we have the following lemma.

Lemma 3 For arbitrary $(\epsilon, s) \in(0,+\infty) \times \mathbb{R}$ and arbitrary $\sigma(>1)$, the operator $L: \mathscr{X}_{\epsilon, s} \rightarrow \mathscr{X}_{\sigma \epsilon, s+1}$ is an isomorphism.

Proof It is obvious that the operator $L$ is linear and surjective from (34). For $q \in \mathscr{X}_{\epsilon, s}$, we have

$$
\begin{aligned}
\|L q\|_{\sigma \epsilon, s+1}^{2} & =|\widehat{L q}(0)|^{2}+\sum_{n \in \mathbb{Z} \backslash\{0\}}|\widehat{L q}(n)|^{2}(\sigma \epsilon)^{2|n|}(2 \pi|n|)^{2(s+1)} \\
& =|\widehat{q}(0)|^{2}+\sum_{n \in \mathbb{Z} \backslash\{0\}}\left|\frac{\sigma^{-|n|}}{4 \pi|n|} \widehat{q}(n)\right|^{2}(\sigma \epsilon)^{2|n|}(2 \pi|n|)^{2(s+1)} \\
& =|\widehat{q}(0)|^{2}+\frac{1}{4} \sum_{n \in \mathbb{Z} \backslash\{0\}}|\widehat{q}(n)|^{2} \epsilon^{2|n|}(2 \pi|n|)^{2 s} .
\end{aligned}
$$

This gives the inequality

$$
\frac{1}{2}\|q\|_{\epsilon, s} \leq\|L q\|_{\sigma \epsilon, s+1} \leq\|q\|_{\epsilon, s}
$$

which shows that the operator $L$ is an isomorphism. 
Remark 5 In [4], the integral operator $L_{\mathrm{C}}$ given by

$$
L_{\mathrm{C}} q(\tau)=-\frac{1}{2 \pi} \int_{S^{1}} \log \left|\rho \mathrm{e}^{2 \pi \mathrm{i} \tau}-\sigma \rho \mathrm{e}^{2 \pi \mathrm{i} \theta}\right| q(\theta) \mathrm{d} \theta
$$

is used instead of $L$ given by (29) for the analysis of the conventional scheme applied to problems in a disk region. The operator $L_{\mathrm{C}}$ is shown to be an isomorphism from $\mathscr{X}_{\epsilon, s}$ to $\mathscr{X}_{\sigma \epsilon, s+1}$ under the somewhat unnatural assumptions that

$$
(\sigma \rho)^{N}-\rho^{N} \neq 1 \text { and } \sigma \rho \neq 1 \text {. }
$$

Lemma 3 for the integral operator $L$ given in this paper does not need these unnatural assumptions.

The following theorem shows the unique solvability of the collocation equation (32) with the Eq. (33) and gives an error estimate of the invariant scheme for the case of a disk region $D_{\rho}$.

Theorem 5 We consider the invariant scheme of the MFS (31) for potential problems (1) in a disk region $D_{\rho}$.

1. The collocation equation (32) with the Eq. (33) have a unique solution $u_{N}^{(\mathrm{I})}$ for arbitrary $(\delta, t) \in(0,+\infty) \times \mathbb{R}$ and arbitrary boundary data $f$ such that $f_{\rho} \in$ $\mathscr{X}_{\delta, t}$.

2. We assume that $(\epsilon, s),(\delta, t) \in(0,+\infty) \times \mathbb{R}$ and $\sigma(>1)$ satisfy

$$
(\delta, t)>\left(1, \frac{1}{2}\right), \quad \max \left\{\frac{\delta}{\sigma^{2}}, \frac{1}{\delta}\right\} \leq \epsilon \leq \min \left\{\delta, \frac{\sigma^{2}}{\delta}\right\}
$$

If $\delta=\epsilon$, we assume $s \leq t$. if $\epsilon=\sigma$, we assume $s<\frac{1}{2}$. Then, if the function $f_{\rho}$ on $S^{1}$ belongs to $\mathscr{X}_{\delta, t}$, we have

$$
\left\|f_{\rho}-\left(u_{N}^{(\mathrm{I})}\right)_{\rho}\right\|_{\epsilon, s} \leq C\left\|f_{\rho}\right\|_{\delta, t} N^{P(\epsilon, s, \delta, t)}\left(\frac{\epsilon}{\delta}\right)^{N / 2}
$$

for sufficiently large $N \in \mathbb{N}$, where $\left(u_{N}^{(\mathrm{I})}\right)_{\rho}$ is the function on $S^{1}$ given by

$$
\left(u_{N}^{(\mathrm{I})}\right)_{\rho}(\tau)=u_{N}^{(\mathrm{I})}\left(\rho \mathrm{e}^{2 \pi \mathrm{i} \tau}\right),
$$

and $P(\epsilon, s, \delta, t)$ is defined by (27).

Theorem 5 is proved by the following lemma.

Lemma 4 1. For arbitrary function $q$ on $S^{1}$, there exists a unique function $q_{N} \in \mathscr{D}_{N}$ such that

$$
L q_{N}=L q \text { on } \Delta_{N}
$$


2. We assume that $(\epsilon, s),(\delta, t) \in(0,+\infty) \times \mathbb{R}$ and $\sigma(>1)$ satisfy

$$
(\delta, t)>\left(\frac{1}{\sigma},-\frac{1}{2}\right), \quad \frac{1}{\sigma^{2}} \max \left\{\delta, \frac{1}{\delta}\right\} \leq \epsilon \leq \min \left\{\delta, \frac{1}{\delta}\right\} .
$$

If $\epsilon=\delta$, we assume $s \leq t$. If $\epsilon=1$, we assume $s<-\frac{1}{2}$. Then, for arbitrary function $q \in \mathscr{X}_{\delta, t}$ and $q_{N} \in \mathscr{D}_{N}$ determined by (35), we have

$$
\left\|q-q_{N}\right\|_{\epsilon, s} \leq C\|q\|_{\delta, t} N^{P_{0}(\epsilon, s, \delta, t)}\left(\frac{\epsilon}{\delta}\right)^{N / 2}
$$

for sufficiently large $N \in \mathbb{N}$, where $P_{0}(\epsilon, s, \delta, t)$ is defined by

$$
P_{0}(\epsilon, s, \delta, t)= \begin{cases}\max \{s-t,-t-1\} & \text { if } \delta \epsilon=\sigma^{-2} \text { and } \sigma^{-2} \leq \delta<1 \\ \max \{s-t, s\} & \text { if } \delta \epsilon=1 \text { and } 1 \leq \delta<\sigma \\ \max \{s-t,-1\} & \text { if } \epsilon=\sigma^{-2} \delta \text { and } 1<\delta<\sigma \\ \max \{s-t,-t-1,-1\} & \text { if }(\delta, \epsilon)=\left(1, \sigma^{-2}\right) \\ \max \{s-t, s,-1\} & \text { if }(\delta, \epsilon)=\left(\sigma, \sigma^{-1}\right) \\ s-t & \text { otherwise. }\end{cases}
$$

Proof of Theorem 5 Since the operator $L: \mathscr{X}_{\sigma^{-1} \delta, t-1} \rightarrow \mathscr{X}_{\delta, t}$ is an isomorphism from Lemma 3, there exists a unique function $q \in \mathscr{X}_{\sigma^{-1} \delta, t-1}$ such that $L q=f_{\rho}$ for $f_{\rho} \in \mathscr{X}_{\delta, t}$. Then, there exists a unique function $q_{N} \in \mathscr{D}_{N}$ such that $L q_{N}=L q=$ $f_{\rho}$ on $\Delta_{N}$ from Lemma 4 , and remarking that the parameters $\left(\sigma^{-1} \epsilon, s-1, \sigma^{-1} \delta, t-1\right)$ satisfy the assumption of Lemma $4,\left(u_{N}^{(\mathrm{I})}\right)_{\rho}=L q_{N}$ satisfies

$$
\begin{aligned}
\left\|f_{\rho}-L q_{N}\right\|_{\epsilon, s} & \leq C\left\|q-q_{N}\right\|_{\sigma^{-1} \epsilon, s-1} \\
& \leq C^{\prime}\|q\|_{\sigma^{-1} \delta, t-1} N^{P_{0}\left(\sigma^{-1} \epsilon, s-1, \sigma^{-1} \delta, t-1\right)}\left(\frac{\epsilon}{\delta}\right)^{N / 2} \\
& \leq C^{\prime \prime}\left\|f_{\rho}\right\|_{\delta, t} N^{P(\epsilon, s, \delta, t)}\left(\frac{\epsilon}{\delta}\right)^{N / 2}
\end{aligned}
$$

where we remark $q_{N} \in \mathscr{X}_{\sigma^{-1} \epsilon, s-1}$ and, then, $q-q_{N} \in \mathscr{X}_{\sigma^{-1} \epsilon, s-1}$ since $\left(\sigma^{-1} \epsilon, s-\right.$ 1) $<(1,-1 / 2)$.

Proof of Lemma 4 We here prove the unique solvability of the collocation equation only, and postpone the estimation of $\left\|q-q_{N}\right\|_{\epsilon, s}$ to the appendix.

We show the unique solvability of the collocation equation $L q_{N}=L q$ on $\Delta_{N}$ by giving the solution explicitly. The collocation equation is equivalent to the equation

$$
C_{0}-\frac{1}{2 \pi} \sum_{j \in \Lambda_{N}} Q_{j} \log \left|\rho-\sigma \rho \omega^{-(i-j)}\right|=L q\left(\frac{i}{N}\right) \quad\left(i \in \Lambda_{N}\right)
$$


if we express $q_{N}$ as (17). Multiplying the both sides by $\omega^{-p i}(p \in \mathbb{Z})$ and taking the sum with respect to $i \in \Lambda_{N}$, we have

$$
\begin{aligned}
C_{0} & =\frac{1}{N} \sum_{i \in \Lambda_{N}} L q\left(\frac{i}{N}\right), \\
\varphi_{p}^{(N)}(\rho) \sum_{j \in \Lambda_{N}} \omega^{-p j} Q_{j} & =\sum_{i \in \Lambda_{N}} \omega^{-p i} L q\left(\frac{i}{N}\right) \quad(p \neq 0 \bmod N),
\end{aligned}
$$

where we used the constraint (8), the formula

$$
\sum_{j \in \Lambda_{N}} \omega^{n j}= \begin{cases}N & \text { if } n \equiv 0 \quad \bmod N \\ 0 & \text { otherwise }\end{cases}
$$

and the function

$$
\begin{aligned}
\varphi_{p}^{(N)}(z)= & -\frac{1}{2 \pi} \sum_{i \in \Lambda_{N}} \omega^{p i} \log \left|z-\sigma \rho \omega^{i}\right| \\
& \left(z \in \mathbb{C} \backslash\left\{\sigma \rho \omega^{i} \mid i \in \Lambda_{N}\right\}, \quad p \in \mathbb{Z}\right) .
\end{aligned}
$$

We can also express the function $\varphi_{p}^{(N)}(z)$ as [5]

$$
\varphi_{p}^{(N)}(z)=\varphi_{p}^{(N)}\left(r \mathrm{e}^{2 \pi \mathrm{i} \tau}\right)= \begin{cases}\frac{N}{4 \pi} \sum_{m \in \mathbb{Z}} \frac{1}{|m|}\left(\frac{r}{\sigma \rho}\right)^{|m|} \mathrm{e}^{2 m \pi \mathrm{i} \tau} & \text { if } p \not \equiv \bmod N \\ -\frac{1}{2 \pi} \log \left|z^{N}-(\sigma \rho)^{N}\right| & \text { if } p \equiv 0 \bmod N\end{cases}
$$

and, especially, we have

$$
\varphi_{p}^{(N)}(\rho)=\frac{N}{4 \pi} \sum_{\substack{m \in \mathbb{Z} \\ m \equiv p}} \frac{\sigma^{-|m|}}{|m|} \neq 0 \text { if } p \not \equiv 0 \quad \bmod N
$$

Then, we can divide the both side of (39) by $\varphi_{p}^{(N)}(\rho)$ and we obtain

$$
\sum_{j \in \Lambda_{N}} \omega^{-p j} Q_{j}=\frac{1}{\varphi_{p}^{(N)}(\rho)} \sum_{i \in \Lambda_{N}} \omega^{-p i} L q\left(\frac{i}{N}\right)
$$

Multiplying the both sides by $\omega^{p k}\left(k \in \Lambda_{N}\right)$ and taking the sum with respect to $p \in \Lambda_{N} \backslash\{0\}$, we obtain

$$
Q_{k}=\frac{1}{N} \sum_{p \in \Lambda_{N} \backslash\{0\}} \sum_{i \in \Lambda_{N}} \omega^{p(k-i)} \frac{L q(i / N)}{\varphi_{p}^{(N)}(\rho)} .
$$


Therefore, we obtain a unique solution $C_{0}$ and $Q_{j}\left(j \in \Lambda_{N}\right)$ of the Eqs. (37) and (8). It means that there exists a unique function $q_{N} \in \mathscr{D}_{N}$ which satisfies the collocation equation $L q_{N}=L q$ on $\Delta_{N}$.

\subsection{Step 2-case of a general region}

We split the integral operator $A$ into $A=L+K$, where $K$ is the integral operator given by (30). Using the function

$$
k(\tau, \theta)=-\frac{1}{2 \pi} \log \left|\frac{\Psi\left(\rho \mathrm{e}^{2 \pi \mathrm{i} \tau}\right)-\Psi\left(\sigma \rho \mathrm{e}^{2 \pi \mathrm{i} \theta}\right)}{\rho \mathrm{e}^{2 \pi \mathrm{i} \tau}-\sigma \rho \mathrm{e}^{2 \pi \mathrm{i} \theta}}\right|,
$$

the operator $K$ is given by

$$
K q(\tau)=\int_{S^{1}} k(\tau, \theta)\{q(\tau)-\widehat{q}(0)\} \mathrm{d} \theta
$$

The Fourier coefficients of $K q$ are given by

$$
\widehat{K q}(n)=\sum_{m \in \mathbb{Z} \backslash\{0\}} \widehat{k}(n, m) \widehat{q}(-m)
$$

where $\widehat{k}(n, m)$ are the Fourier coefficients of $k(\tau, \theta)$, that is,

$$
\widehat{k}(n, m)=\iint_{S^{1} \times S^{1}} k(\tau, \theta) \mathrm{e}^{-2 \pi \mathrm{i}(n \tau+m \theta)} \mathrm{d} \tau \mathrm{d} \theta .
$$

The following lemma shows the compactness of the operator $K$.

Lemma 5 We assume the following conditions.

1. The conformal mapping $\Psi:\{w \in \mathbb{C}|| w \mid>\rho\} \rightarrow \mathbb{C} \backslash \bar{\Omega}$ admits a conformal extension to $\{w \in \mathbb{C}|| w \mid \geq \alpha \rho\}(0<\alpha<1)$.

2. The parameters $(\epsilon, s),(\delta, t) \in(0,+\infty) \times \mathbb{R}, \alpha$ and $\sigma(>1)$ satisfy

$$
(\epsilon, s)>\left(\frac{\alpha}{\sigma}, \frac{1}{2}\right) \text { and }(\delta, t)<\left(\frac{1}{\alpha},-\frac{1}{2}\right) \text {, }
$$

Then, the operator $K: \mathscr{X}_{\epsilon, s} \longrightarrow \mathscr{X}_{\delta, t}$ is compact.

We can prove this lemma in the same way as the proof of Lemma 4.3 of [4].

From the above lemma, we can regard the operator $A$ as a perturbation of the isomorphism $L$ by the compact operator $K$. Then, $A$ is a Fredholm operator and is shown to be an isomorphism by the Riesz-Schauder theory as in the following lemma. 
Lemma 6 We assume the following conditions.

1. The conformal mapping $\Psi:\{w \in \mathbb{C}|| w \mid>\rho\}$ admits a conformal extension to $\{w \in \mathbb{C}|| w \mid \geq \alpha \rho\}(0<\alpha<1)$.

2. The parameters $(\epsilon, s),(\delta, t) \in(0,+\infty) \times \mathbb{R}, \alpha$ and $\sigma(>1)$ satisfy

$$
\left(\frac{\alpha}{\sigma}, \frac{1}{2}\right)<(\epsilon, s)<\left(\frac{1}{\sigma \alpha},-\frac{3}{2}\right) .
$$

Then, the following (i) and (ii) hold true.

(i) The operator $A: \mathscr{X}_{\epsilon, s} \rightarrow \mathscr{X}_{\sigma \epsilon, s+1}$ is bounded.

(ii) Moreover if $\alpha \sigma<1$, then the operator $A$ is an isomorphism.

Proof (i) The operator $L: \mathscr{X}_{\epsilon, s} \rightarrow \mathscr{X}_{\sigma \epsilon, s+1}$ is bounded from Lemma 3 and the operator $K: \mathscr{X}_{\epsilon, s} \rightarrow \mathscr{X}_{\sigma \epsilon, s+1}$ is compact, that is, it is bounded from Lemma 5, whose assumptions are satisfied since $(\sigma \epsilon, s+1)<\left(\alpha^{-1},-1 / 2\right)$. Therefore, the operator $A=L+K: \mathscr{X}_{\epsilon, s} \rightarrow \mathscr{X}_{\sigma \epsilon, s+1}$ is bounded.

(ii) Since the operator $A=L+K$ is a Fredholm operator of index 0 , we only have to prove that $A$ is injective. We assume that $q \in \mathscr{X}_{\epsilon, s}$ satisfies $A q=0$. Then, we have $q=-L^{-1} K q$. From Lemma 5, $K q \in \mathscr{X}_{\alpha^{-1}, t}$ for arbitrary $t<-1 / 2$ and $q=-L^{-1} K q \in \mathscr{X}_{(\sigma \alpha)^{-1}, t-1}$. Since $\sigma \alpha<1, q$ is analytic and, then, we have $q=0$ from the lemma below.

Lemma 7 Let $\Gamma$ be a smooth closed Jordan curve in $\mathbb{C}$ and $q: S^{1} \rightarrow \mathbb{R}$ be a Hölder continuous function. Then, if $A q=0$, we have $q=0$.

Proof We here use the notations

$$
\begin{aligned}
\zeta(\theta) & =\Psi\left(\sigma \rho \mathrm{e}^{2 \pi \mathrm{i} \theta}\right) \quad\left(\theta \in S^{1}\right), \\
\Gamma_{\sigma \rho} & =\left\{\zeta(\theta) \mid \theta \in S^{1}\right\}, \\
\Omega_{\sigma \rho} & \left.=\text { (the interior of } \Gamma_{\sigma \rho}\right), \quad \Omega_{\sigma \rho}^{\prime}=\left(\text { the exterior of } \Gamma_{\sigma \rho}\right) .
\end{aligned}
$$

We define the function $u(z)(z \in \mathbb{C})$ by

$$
u(z)=\widehat{q}(0)-\frac{1}{2 \pi} \int_{S^{1}} \log |z-\zeta(\theta)|\{q(\theta)-\widehat{q}(0)\} \mathrm{d} \theta .
$$

The function $u(z)$ is harmonic in $\mathbb{C} \backslash \Gamma_{\sigma \rho}$ and continuous in $\mathbb{C}$ by Theorem $15.8 \mathrm{~b}$ of [2]. From the assumption, we have $u=0$ on $\Gamma$ and, then, $u=0$ in $\Omega$ by the principle of the maximum for harmonic functions. We let $v$ be the conjugate harmonic function of $u$

$$
v(z)=-\frac{1}{2 \pi} \int_{S^{1}} \arg \{z-\zeta(\theta)\}\{q(\theta)-\widehat{q}(0)\} \mathrm{d} \theta
$$


and put $f=u+\mathrm{i} v$. Since $u=0$ in $\Omega$, we have $v \equiv c_{1}$ (const.) in $\Omega$. Then, $f$ is an analytic function in $\Omega_{\sigma \rho}$ and $f \equiv \mathrm{i} c_{1}$ in $\Omega$. It follows that $f \equiv \mathrm{i} c_{1}$ in $\Omega_{\sigma \rho}$ by the identity theorem and, then, $u \equiv 0$ in $\Omega_{\sigma \rho}$. From the continuity of $u$ in $\mathbb{C}$, we have $u=0$ on $\Gamma_{\sigma \rho}$.

The function $u$ is harmonic in $\Omega_{\sigma \rho}^{\prime}$, continuous in $\Omega_{\sigma \rho}^{\prime} \cup \Gamma_{\sigma \rho}$ and $u=0$ on $\Gamma_{\sigma \rho}$. Then, the function $u(\Psi(w))$ is harmonic in $\{w \in \mathbb{C}|| w \mid>\sigma \rho\}$, continuous in $\{w \in \mathbb{C}|| w \mid \geq \sigma \rho\}$ and satisfies the boundary condition $u(\Psi(w))=0$ on $\{w \in$ $\mathbb{C}|| w \mid=\sigma \rho\}$. Therefore, $u(\Psi(w))=0$ in $\{w \in \mathbb{C}|| w \mid \geq \sigma \rho\}$, and, then, $u(z)=0$ in $\Omega_{\sigma \rho}^{\prime} \cup \Gamma_{\sigma \rho}$. Consequently, the function $f$ is analytic in $\mathbb{C} \backslash \Gamma_{\sigma \rho}$ and

$$
f(z)=\widehat{q}(0)-\frac{1}{2 \pi} \int_{S^{1}} \log \{z-\zeta(\theta)\}\{q(\theta)-\widehat{q}(0)\} \mathrm{d} \theta=\left\{\begin{array}{l}
\mathrm{i} c_{1} \text { in } \Omega_{\sigma \rho} \\
\mathrm{i} c_{2} \text { in } \Omega_{\sigma \rho}^{\prime},
\end{array}\right.
$$

where $c_{2}$ is a real constant. Differentiating it with respect to $z$, we have

$$
\frac{1}{2 \pi} \int_{S^{1}} \frac{q(\theta)-\widehat{q}(0)}{\zeta(\theta)-z} \mathrm{~d} \theta=0 \text { in } \mathbb{C} \backslash \Gamma_{\sigma \rho}=\Omega_{\sigma \rho} \cup \Omega_{\sigma \rho}^{\prime} .
$$

Introducing the function

$$
\widetilde{q}(\zeta(\theta))=\frac{\mathrm{i}(q(\theta)-\widehat{q}(0))}{\zeta^{\prime}(\theta)}
$$

we have

$$
p(z) \equiv \frac{1}{2 \pi \mathrm{i}} \int_{\Gamma_{\sigma \rho}} \frac{\widetilde{q}(\zeta)}{\zeta-z} \mathrm{~d} \zeta=0 \text { in } \mathbb{C} \backslash \Gamma_{\sigma \rho}
$$

For $z \in \Gamma_{\sigma \rho}$,

$$
p_{+}(z) \equiv \lim _{\substack{\zeta \rightarrow z \\ \zeta \in \Omega_{\sigma \rho}}} p(\zeta)=0 \text { and } p_{-}(z) \equiv \lim _{\substack{\zeta \rightarrow z \\ \zeta \in \Omega_{\sigma \rho}^{\prime}}} p(\zeta)=0,
$$

and we have

$$
\widetilde{q}(z)=p_{+}(z)-p_{-}(z)=0 \quad\left(z \in \Gamma_{\sigma \rho}\right)
$$

from Sokhotskyi's formula [2] and $q(\theta)-\widehat{q}(0)=0 \quad\left(\theta \in S^{1}\right)$. Substituting it into (44), we have

$$
f(z)=\widehat{q}(0)=\left\{\begin{array}{l}
\mathrm{i} c_{1} \text { in } \Omega_{\sigma \rho} \\
\mathrm{i} c_{2} \text { in } \Omega_{\sigma \rho}^{\prime}
\end{array}\right.
$$

and $\widehat{q}(0)=c_{1}=c_{2}=0$ since $\widehat{q}(0), c_{1}$ and $c_{2}$ are real constants. Therefore, we have $q(\theta)=\widehat{q}(0)+\{q(\theta)-\widehat{q}(0)\}=0\left(\theta \in S^{1}\right)$. 
Lemma 8 We assume that the conformal mapping $\Psi$ admits a conformal extension to $\{w \in \mathbb{C}|| w \mid \geq \alpha \rho\}(0<\alpha<1)$, and the parameters $(\epsilon, s),(\delta, t) \in(0,+\infty) \times \mathbb{R}$, $\alpha$ and $\sigma(>1)$ satisfy the following conditions.

1. $\left(\frac{1}{\sigma},-\frac{1}{2}\right)<(\delta, t)<\left(\frac{1}{\sigma \alpha},-\frac{3}{2}\right)$.

2. $\frac{1}{\sigma^{2}} \max \left\{\delta, \frac{1}{\delta}\right\} \leq \epsilon \leq \min \left\{\delta, \frac{1}{\delta}\right\}$. If $\epsilon=1$, we assume $s<-1 / 2$. If $\epsilon=\delta$, we assume $s<t$.

3. $\sigma \alpha<1$.

4. $\left(\frac{\alpha}{\sigma}, \frac{1}{2}\right)<(\epsilon, s)$.

Then, the following (i) and (ii) hold true for sufficiently large $N \in \mathbb{N}$.

(i) The collocation equation for $q_{N} \in \mathscr{D}_{N}$

$$
A q_{N}=A q \text { on } \Delta_{N}
$$

where $q$ is a given function of $\mathscr{X}_{\delta, t}$, has a unique solution $q_{N}$.

(ii) There exists a constant $C>0$ such that, for arbitrary $q \in \mathscr{X}_{\delta, t}$ and $q_{N} \in \mathscr{D}_{N}$ determined by (45) we have

$$
\left\|q-q_{N}\right\|_{\epsilon, s} \leq C\|q\|_{\delta, t} N^{P_{0}(\epsilon, s, \delta, t)}\left(\frac{\epsilon}{\delta}\right)^{N / 2} .
$$

where $P_{0}(\epsilon, s, \delta, t)$ is defined by (36).

Proof (i) If we write $q_{N} \in \mathscr{D}_{N}$ as (17), the collocation equation (45) is equivalent to the system of linear equations for $C_{0}$ and $Q_{j}\left(j \in \Lambda_{N}\right)$

$$
\mathrm{G} Q=f
$$

with

$$
\begin{aligned}
& \mathbf{G}=\left[\begin{array}{l|l}
{\left[G_{i j}\right]_{i, j \in \Lambda_{N}}} & \vdots \\
\hline 1
\end{array}\right], \quad G_{i j}=-\frac{1}{2 \pi} \log \left|\Psi\left(\rho \omega^{i}\right)-\Psi\left(\sigma \rho \omega^{j}\right)\right|, \\
& \hline \boldsymbol{Q}=\left[\frac{\left[Q_{i}\right]_{i \in \Lambda_{N}}}{C_{0}}\right] \text { and } \boldsymbol{f}=\left[\frac{[A q(i / N)]_{i \in \Lambda_{N}}}{0}\right] .
\end{aligned}
$$

Then, we have

(The collocation equation (45) is uniquely solvable.)

$\Longleftrightarrow$ (The coefficient matrix $\mathrm{G}$ of the linear system(46) is regular.)

$\Longleftrightarrow$ (If $\mathrm{G} Q=\mathbf{0}$, then $\boldsymbol{Q}=\mathbf{0}$.)

$\Longleftrightarrow$ (If $q_{N} \in \mathscr{D}_{N}$ and $A q_{N}=0$ on $\Delta_{N}$, then $q_{N}=0$.). 
Therefore, we only have to show that, if $q_{N} \in \mathscr{D}_{N}$ and $A q_{N}=0$ on $\Delta_{N}$, we have $q_{N}=0$.

The operator $L: \mathscr{X}_{\epsilon, s} \rightarrow \mathscr{X}_{\sigma \epsilon, s+1}$ is an isomorphism by Lemma 3 and the operator $A: \mathscr{X}_{\epsilon, s} \rightarrow \mathscr{X}_{\sigma \epsilon, s+1}$ is an isomorphism from Lemma 6, whose assumptions are satisfied since $(\alpha / \sigma, 1 / 2)<(\epsilon, s)<\left((\sigma \alpha)^{-1},-3 / 2\right)$. Then, we have

$$
\left\|q_{N}\right\|_{\epsilon, s} \leq C\left\|A q_{N}\right\|_{\sigma \epsilon, s+1} \leq C^{\prime}\left\|L^{-1} A q_{N}\right\|_{\epsilon, s},
$$

where we remark $q_{N} \in \mathscr{X}_{\epsilon, s}$ from $(\epsilon, s)<(1,-1 / 2)$ and Lemma 2. We now let

$$
w_{0}=q_{N}-L^{-1} A q_{N},
$$

which belongs to $\mathscr{X}_{\epsilon, s}$ and satisfies that

$$
\begin{aligned}
& L^{-1} A q_{N}=q_{N}-w_{0}, \\
& L q_{N}=L w_{0} \text { on } \Delta_{N}, \\
& w_{0}=L^{-1}(L-A) q_{N} .
\end{aligned}
$$

Since $q_{N}$ satisfies the collocation equation (48), we have

$$
\left\|L^{-1} A q_{N}\right\|_{\epsilon, s}=\left\|q_{N}-w_{0}\right\|_{\epsilon, s} \leq C\left\|w_{0}\right\|_{\delta, t} N^{P_{0}(\epsilon, s, \delta, t)}\left(\frac{\epsilon}{\delta}\right)^{N / 2}
$$

if $w_{0} \in \mathscr{X}_{\delta, t}$ from Lemma 4 , whose assumptions are satisfied from the assumptions of this lemma. The operator $L: \mathscr{X}_{\delta, t} \rightarrow \mathscr{X}_{\sigma \delta, t+1}$ is an isomorphism from Lemma 3 and the operator $L-A=-K: \mathscr{X}_{\epsilon, s} \rightarrow \mathscr{X}_{\sigma \delta, t+1}$ is compact and, then, it is bounded from Lemma 5, whose assumptions are satisfied since $(\epsilon, s)>(\alpha / \sigma, 1 / 2)$ and $(\sigma \delta, t+1)<\left(\alpha^{-1},-1 / 2\right)$. Then, we have $w_{0}=L^{-1}(L-A) q_{N} \in \mathscr{X}_{\delta, t}$ and

$$
\left\|w_{0}\right\|_{\delta, t}=\left\|L^{-1}(L-A) q_{N}\right\|_{\delta, t} \leq C\left\|(L-A) q_{N}\right\|_{\sigma \delta, t+1} \leq C^{\prime}\left\|q_{N}\right\|_{\epsilon, s} .
$$

Gathering the above three inequalities, we have

$$
\left\|q_{N}\right\|_{\epsilon, s} \leq C\left\|q_{N}\right\|_{\epsilon, s} N^{P_{0}(\epsilon, s, \delta, t)}\left(\frac{\epsilon}{\delta}\right)^{N / 2}
$$

and, then,

$$
\left\{1-C N^{P_{0}(\epsilon, s, \delta, t)}\left(\frac{\epsilon}{\delta}\right)^{N / 2}\right\}\left\|q_{N}\right\|_{\epsilon, s} \leq 0 .
$$

We here remark that

$$
N^{P_{0}(\epsilon, s, \delta, t)}\left(\frac{\epsilon}{\delta}\right)^{N / 2}=\mathrm{o}(1) \quad \text { as } N \rightarrow \infty
$$


In fact, we have $\epsilon / \delta \leq 1$ and, if $\epsilon / \delta=1$, we have

$$
\begin{aligned}
P_{0}(\epsilon, s, \delta, t) & = \begin{cases}\max \{s-t,-t-1\} & \text { if }(\delta, \epsilon)=\left(\sigma^{-1}, \sigma^{-1}\right) \\
\max \{s-t, s\} & \text { if }(\delta, \epsilon)=(1,1) \\
s-t & \text { otherwise }\end{cases} \\
& <0 .
\end{aligned}
$$

Therefore, for sufficiently large $N \in \mathbb{N}$, we have $\left\|q_{N}\right\|_{\epsilon, s}=0$ and $q_{N}=0$.

(ii) We have a unique solution $q_{N} \in \mathscr{D}_{N}$ of the collocation equation $A q_{N}=A q$ on $\Delta_{N}$ for arbitrary $q \in \mathscr{X}_{\delta, t}$ by the former part of this proof. We remark that $q \in$ $\mathscr{X}_{\epsilon, s}$ since $(\epsilon, s)<(\delta, t)$ and, then, $q_{N}-q \in \mathscr{X}_{\epsilon, s}$ since $q_{N} \in \mathscr{X}_{\epsilon, s}$ due to $(\epsilon, s)<(1,-1 / 2)$ and Lemma 2. Since the operators $L: \mathscr{X}_{\epsilon, s} \rightarrow \mathscr{X}_{\sigma \epsilon, s+1}$ and $A: \mathscr{X}_{\epsilon, s} \rightarrow \mathscr{X}_{\sigma \epsilon, s+1}$ are already shown to be isomorphic, we have

$$
\left\|q_{N}-q\right\|_{\epsilon, s} \leq C\left\|A\left(q_{N}-q\right)\right\|_{\sigma \epsilon, s+1} \leq C^{\prime}\left\|L^{-1} A\left(q_{N}-q\right)\right\|_{\epsilon, s} .
$$

Now we let

$$
w=q_{N}-L^{-1} A\left(q_{N}-q\right),
$$

which belongs to $\mathscr{X}_{\epsilon, s}$ and satisfies that

$$
\begin{aligned}
& L^{-1} A\left(q_{N}-q\right)=q_{N}-w, \\
& L q_{N}=L w \text { on } \Delta_{N}, \\
& w=q+L^{-1}(L-A)\left(q_{N}-q\right) .
\end{aligned}
$$

Since $q_{N}$ satisfies the collocation equation (49), we have

$$
\left\|L^{-1} A\left(q_{N}-q\right)\right\|_{\epsilon, s}=\left\|q_{N}-w\right\|_{\epsilon, s} \leq C\|w\|_{\delta, t} N^{P_{0}(\epsilon, s, \delta, t)}\left(\frac{\epsilon}{\delta}\right)^{N / 2},
$$

if $w \in \mathscr{X}_{\delta, t}$ from Lemma 4 , whose assumptions are satisfied from the assumptions of this lemma. Since the operator $L-A=-K: \mathscr{X}_{\epsilon, s} \rightarrow \mathscr{X}_{\sigma \delta, t+1}$ is already shown to be compact and, then, bounded, we have $w=q+L^{-1}(L-A)\left(q_{N}-q\right) \in$ $\mathscr{X}_{\delta, t}$ and

$$
\begin{aligned}
\|w\|_{\delta, t} & =\left\|q+L^{-1}(L-A)\left(q_{N}-q\right)\right\|_{\delta, t} \\
& \leq\|q\|_{\delta, t}+\left\|L^{-1}(L-A)\left(q_{N}-q\right)\right\|_{\delta, t} \\
& \leq\|q\|_{\delta, t}+C\left\|(L-A)\left(q_{N}-q\right)\right\|_{\sigma \delta, t+1} \\
& \leq\|q\|_{\delta, t}+C^{\prime}\left\|q_{N}-q\right\|_{\epsilon, s} .
\end{aligned}
$$

Gathering the above three inequalities, we have

$$
\left\|q_{N}-q\right\|_{\epsilon, s} \leq C N^{P_{0}(\epsilon, s, \delta, t)}\left(\frac{\epsilon}{\delta}\right)^{N / 2}\left(\|q\|_{\delta, t}+\left\|q_{N}-q\right\|_{\epsilon, s}\right) .
$$


Since $N^{P_{0}(\epsilon, s, \delta, t)}(\epsilon / \delta)^{N / 2}=\mathrm{o}(1)$ as $N \rightarrow \infty$ from the discussion of the former part of this proof, we have

$$
\left\|q_{N}-q\right\|_{\epsilon, s} \leq C^{\prime}\|q\| \delta, t N^{P_{0}(\epsilon, s, \delta, t)}\left(\frac{\epsilon}{\delta}\right)^{N / 2}
$$

Proof of Theorem 4 (i) Let $\widetilde{f} \in \mathscr{X}_{\delta, t}$. Then, there exists a unique function $q \in$ $\mathscr{X}_{\sigma^{-1} \delta, t-1}$ such that $A q=\widetilde{f}$ from Lemma 6, whose assumptions are satisfied by

$$
\left(\frac{\alpha}{\sigma}, \frac{1}{2}\right)<\left(\frac{1}{\sigma},-\frac{1}{2}\right)<\left(\frac{\delta}{\sigma}, t-1\right)<\left(\frac{1}{\sigma \alpha},-\frac{3}{2}\right)
$$

and $\sigma \alpha<1$. For this $q$, there exists a unique function $q_{N} \in \mathscr{D}_{N}$ such that $A q_{N}=A q$ on $\Delta_{N}$ from Lemma 8, whose assumptions are satisfied for $(\epsilon / \sigma, s-1)$ and $(\delta / \sigma, t-1)$ by the following.

$$
-\left(\frac{1}{\sigma},-\frac{1}{2}\right)<\left(\frac{\delta}{\sigma}, t-1\right)<\left(\frac{1}{\sigma \alpha},-\frac{3}{2}\right) .
$$

$-\frac{1}{\sigma^{2}} \max \left\{\frac{\delta}{\sigma}, \frac{\sigma}{\delta}\right\} \leq \frac{\epsilon}{\sigma} \leq \min \left\{\frac{\delta}{\sigma}, \frac{\sigma}{\delta}\right\}$. If $\epsilon / \sigma=\delta / \sigma$, we further assume $s-1<t-1$. If $\epsilon / \sigma=\delta / \sigma=1$, we further assume $s-1<-1 / 2$.

$-\sigma \alpha<1$.

$-\left(\frac{\alpha}{\sigma}, \frac{1}{2}\right)<\left(\frac{\epsilon}{\sigma}, s-1\right)$.

It means that there exists a unique function $q_{N} \in \mathscr{D}_{N}$ such that $A q_{N}=\tilde{f}$ on $\Delta_{N}$.

(ii) First, we remark that $q-q_{N} \in \mathscr{X}_{\sigma^{-1} \epsilon, s-1}$ from $(\epsilon / \sigma, s-1)<(\delta / \sigma, t-1)$ and Lemma 1. Since the operator $A: \mathscr{X}_{\epsilon / \sigma, s-1} \rightarrow \mathscr{X}_{\epsilon, s}$ is an isomorphism from Lemma 6, whose assumptions are satisfied by

$$
\left(\frac{\alpha}{\sigma}, \frac{1}{2}\right)<\left(\frac{\epsilon}{\sigma}, s-1\right)<\left(\frac{\delta}{\sigma}, t-1\right)<\left(\frac{1}{\sigma \alpha},-\frac{3}{2}\right)
$$

and $\sigma \alpha<1$, we have

$$
\begin{aligned}
\left\|\tilde{f}-A q_{N}\right\|_{\epsilon, s} & =\left\|A\left(q-q_{N}\right)\right\|_{\epsilon, s} \leq C\left\|q-q_{N}\right\|_{\sigma^{-1} \epsilon, s-1} \\
& \leq C^{\prime}\|q\|_{\sigma^{-1} \delta, t-1} N^{P_{0}\left(\sigma^{-1} \epsilon, s-1, \sigma^{-1} \delta, t-1\right)}\left(\frac{\epsilon}{\delta}\right)^{N / 2} \\
& \leq C^{\prime \prime}\|A q\|_{\delta, t} N^{P(\epsilon, s, \delta, t)}\left(\frac{\epsilon}{\delta}\right)^{N / 2} \\
& =C^{\prime \prime}\|\widetilde{f}\|_{\delta, t} N^{P(\epsilon, s, \delta, t)}\left(\frac{\epsilon}{\delta}\right)^{N / 2} .
\end{aligned}
$$


Here, we used the fact that $A: \mathscr{X}_{\sigma^{-1} \delta, t-1} \rightarrow \mathscr{X}_{\delta, t}$ is an isomorphism on the first and third inequalities, which holds true by the discussion in the former part of this proof, and we used Lemma 8 on the second inequality, whose assumptions are satisfied by the discussion in the former part of this proof. Therefore, we obtain the theorem.

\section{Concluding remarks}

In this paper, we proposed to position the charge points and the collocation points of the invariant scheme of the MFS using a conformal mapping of the exterior of a disk to the exterior of the problem region as Katsurada proposed for the conventional scheme of the MFS. A theoretical analysis shows exponential convergence of the invariant scheme, which resemble that of the conventional scheme. It is remarkable that the convergence theorem of the invariant scheme does not need the unnatural assumptions which are needed in the convergence theorem of the conventional scheme. This may be a reflection of the fact that the invariant scheme has the invariance properties under the trivial affine transformations.

The results of this paper give the error estimate of the invariant scheme only on the boundary of the problem region and does not give an error estimate on the whole region. It seems difficult to give an error estimate in the interior of the region. The reason is that we estimate the error using the exterior conformal mapping which gives the charge points and the collocation points. We reduce our problem to the approximation error estimate of the function $u\left(\Psi\left(\rho \mathrm{e}^{2 \pi \mathrm{i} \tau}\right)\right)$ on $S^{1}$, but the mapping function $\Psi$ cannot be extended to the whole disk $\{w \in \mathbb{C}|| w \mid<\rho\}$, that is, the range of the conformal mapping $\Psi$ cannot cover the whole region $\Omega$. Thus, it is difficult to estimate the error in the interior of the region using the exterior conformal mapping. It may be possible to estimate the error on the whole region if we use an interior mapping function $\Phi:\{w \in \mathbb{C}|| w \mid<\rho\} \rightarrow \Omega$, which admits a conformal extension to a disk $\{w \in \mathbb{C}|| w \mid \leq \beta \rho\}$ with $\beta>1$ as in Katsurada's study for the conventional scheme [3].

From practical point of view, exterior conformal mappings are useful compared with interior conformal mappings. It is because we can position the charge points freely in the exterior of the problem region using a exterior conformal mapping while the area where the charge points can be positioned is restricted using an interior conformal mapping.

\section{Appendix: Proof of Lemma 4-estimation of $\left\|q-q_{N}\right\|_{\epsilon, s}$}

First, we rewrite the expression of the $C_{0}$ and $Q_{j}\left(j \in \Lambda_{N}\right)$ given by (38) and (43) Substituting (38) and (43) into

$$
\widehat{q}_{N}(n)=C_{0} \delta_{n 0}+\sum_{j \in \Lambda_{N}} Q_{j} \omega^{-n j}
$$


and using the formula (40) and the expression (34) of $L q$, we have

$$
\widehat{q}_{N}(n)=\left\{\begin{array}{lll}
\frac{N}{4 \pi \varphi_{n}^{(N)}(\rho)} \sum_{m \in \mathbb{Z}} \frac{\sigma^{-|m|}}{|m|} \widehat{q}(m) & \text { if } n \not \equiv 0 \quad \bmod N \\
\widehat{q}(0)+\frac{1}{4 \pi} \sum_{\substack{m \in \mathbb{Z} \backslash\{0\} \\
m \equiv 0}} \frac{\sigma^{-|m|}}{|m|} \widehat{q}(m) & \text { if } n=0 \\
0 & \text { if } n \equiv 0 \quad \bmod N \text { and } n \neq 0 .
\end{array}\right.
$$

Then, we have

$$
\begin{aligned}
\left\|q-q_{N}\right\|_{\epsilon, s}^{2}= & \left|\widehat{q}(0)-\widehat{q}_{N}(0)\right|^{2}+\sum_{n \neq 0}\left|\widehat{q}(n)-\widehat{q}_{N}(n)\right|^{2} \epsilon^{2|n|}(2 \pi|n|)^{2 s} \\
\leq & \left|\widehat{q}(0)-\widehat{q}_{N}(0)\right|^{2}+(2 \pi)^{2 s}\left\{2 \sum_{n \in \mathbb{Z} \backslash \Lambda_{N}}|\widehat{q}(n)|^{2} \epsilon^{2|n|}|n|^{2 s}\right. \\
& \left.+2 \sum_{n \in \mathbb{Z} \backslash \Lambda_{N}}\left|\widehat{q}_{N}(n)\right|^{2} \epsilon^{2|n|}|n|^{2 s}+\sum_{n \in \Lambda_{N} \backslash\{0\}}\left|\widehat{q}(n)-\widehat{q}_{N}(n)\right|^{2} \epsilon^{2|n|}|n|^{2 s}\right\} \\
\equiv & T_{1}+(2 \pi)^{2 s}\left(2 T_{2}+2 T_{3}+T_{4}\right) .
\end{aligned}
$$

The terms $T_{1}, T_{2}$ and $T_{3}$ are estimated in the same way as [4] as follows. Regarding $T_{1}$, we have

$$
\widehat{q}_{N}(0)-\widehat{q}(0)=\frac{1}{4 \pi} \sum_{\substack{m \equiv 0 \\ m \neq 0}} \frac{\sigma^{-|m|}}{|m|} \widehat{q}(m),
$$

and

$$
\begin{aligned}
T_{1} & =\frac{1}{(4 \pi)^{2}}\left\{\sum_{m \equiv 0 m \neq 0}|\widehat{q}(m)| \delta^{|m|} \underline{m}^{t} \cdot \frac{|m|^{-(t+1)}}{(2 \pi)^{t}}(\delta \sigma)^{-|m|}\right\}^{2} \\
& \leq C_{t}\left\{\sum_{\substack{m \equiv 0 \\
m \neq 0}}|\widehat{q}(m)|^{2} \delta^{2|m|} \underline{m}^{2 t}\right\}\left\{\sum_{\begin{array}{l}
m \equiv 0 \\
m \neq 0
\end{array}}|m|^{-2(t+1)}(\sigma \delta)^{-2|m|}\right\} \\
& \leq C_{\sigma \delta, t}\|q\|_{\delta, t}^{2} N^{-2(t+1)}(\sigma \delta)^{-2 N} \\
& \leq C_{\sigma \delta, t}^{\prime}\|q\|_{\delta, t}^{2}\left(\frac{\epsilon}{\delta}\right)^{N} \times \begin{cases}N^{-2(t+1)} & \text { if } \delta \epsilon=\sigma^{-2} \text { and } s \leq-1 \\
N^{2(s-t)} & \text { otherwise, }\end{cases}
\end{aligned}
$$

where we remark that the underlined sum is convergent since $(\sigma \delta, t+1)>(1,1 / 2)$ and we used $(\sigma \delta)^{-2} \leq \epsilon / \delta\left(\Leftrightarrow \delta \epsilon \geq \sigma^{-2}\right)$ on the third inequality. 
Regarding $T_{2}$, we have

$$
\begin{aligned}
T_{2} & =\sum_{n \in \mathbb{Z} \backslash \Lambda_{N}}|\widehat{q}(n)|^{2} \delta^{2|n|}(2 \pi|n|)^{2 t} \cdot \frac{|n|^{2(s-t)}}{(2 \pi)^{2 t}}\left(\frac{\epsilon}{\delta}\right)^{2|n|} \\
& \leq \frac{N^{2(s-t)}}{(2 \pi)^{2 t}}\left(\frac{\epsilon}{\delta}\right)^{N} \sum_{n \in \mathbb{Z} \backslash \Lambda_{N}}|\widehat{q}(n)|^{2} \delta^{2|n|} \underline{n}^{2 t} \cdot\left\{\left(\frac{|n|}{N}\right)^{2(s-t)}\left(\frac{\epsilon}{\delta}\right)^{2|n|-N}\right\} \\
& \leq \frac{N^{2(s-t)}}{(2 \pi)^{2 t}}\left(\frac{\epsilon}{\delta}\right)^{N}\|q\|_{\delta, t}^{2} \times \sup _{n \in \mathbb{Z} \backslash \Lambda_{N}}\left\{\left(\frac{|n|}{N}\right)^{2(s-t)}\left(\frac{\epsilon}{\delta}\right)^{2|n| / N-1}\right\} \\
& \leq C_{\epsilon / \delta, s, t}\|q\|_{\delta, t} N^{2(s-t)}\left(\frac{\epsilon}{\delta}\right)^{N},
\end{aligned}
$$

where we used $\epsilon / \delta \leq 1$ on the second inequality and we remark that the underlined supremum is finite since $(\epsilon / \delta, s-t) \leq(1,0)$.

Regarding $T_{3}$, we have

$$
\begin{aligned}
T_{3} & =\sum_{p \in \Lambda_{N} \backslash\{0\}} \sum_{\substack{l \in \mathbb{Z} \\
l \neq 0}}\left|\widehat{q}_{N}(l N+p)\right|^{2} \epsilon^{2|l N+p|}|l N+p|^{2 s} \\
& =\sum_{p \in \Lambda_{N} \backslash\{0\}}\left|\widehat{q}_{N}(p)\right|^{2} \sum_{l \neq 0} \epsilon^{2|l N+p|}|l N+p|^{2 s} \\
& \leq C_{\epsilon, s} N^{2 s} \epsilon^{2 N} \sum_{p \in \Lambda_{N} \backslash\{0\}} \epsilon^{-2|p|}\left|\widehat{q}_{N}(p)\right|^{2},
\end{aligned}
$$

where we remark that $\widehat{q}_{N}(n)=0$ if $n \equiv 0 \bmod N$ and $n \neq 0$ on the first equality and the underlined sum is convergent by $(\epsilon, s)<(1,-1 / 2)$, and

$$
\begin{aligned}
\left|\widehat{q}_{N}(p)\right|^{2} & \leq\left\{\frac{N}{4 \pi \varphi_{p}^{(N)}(\rho)}\right\}^{2}\left\{\sum_{m \equiv p} \frac{\sigma^{-|m|}}{|m|}|\widehat{q}(m)|\right\}^{2} \\
& \leq|p|^{2} \sigma^{2|p|}\left\{\sum_{m \equiv p}|\widehat{q}(m)| \delta^{|m|} \underline{m}^{t} \cdot \frac{|m|^{-(t+1)}}{(2 \pi)^{t}}(\sigma \delta)^{-|m|}\right\}^{2} \\
& \leq \frac{|p|^{2} \sigma^{2|p|}}{(2 \pi)^{2 t}}\left\{\sum_{m \equiv p}|\widehat{q}(m)|^{2} \delta^{2|m|} \underline{m}^{2 t}\right\}\{\underbrace{\left\{\sum_{m \equiv p}|m|^{-2(t+1)}(\sigma \delta)^{-2|m|}\right\}},
\end{aligned}
$$

where we used $\varphi_{p}^{(N)}(\rho) \geq \frac{N}{4 \pi} \frac{\sigma^{-|p|}}{|p|}$ obtained from (42) on the second inequality. Since the sum (a) is convergent by $(\sigma \delta, t)>(1,-1 / 2)$ and estimated as 


$$
\begin{aligned}
\text { (a) } & =\sum_{l \in \mathbb{Z}}|l N+p|^{-2(t+1)}(\sigma \delta)^{-2|l N+p|} \\
& =|p|^{-2(t+1)}(\sigma \delta)^{-2|p|}+\sum_{l \neq 0}|l N+p|^{-2(t+1)}(\sigma \delta)^{-2|l N+p|} \\
& \leq|p|^{-2(t+1)}(\sigma \delta)^{-2|p|}+C_{\sigma \delta} N^{-2(t+1)}(\sigma \delta)^{-2(N-|p|)},
\end{aligned}
$$

we have

$$
\begin{aligned}
T_{3} \leq & C_{\epsilon, s, t} N^{2 s} \epsilon^{2 N} \sum_{p \in \Lambda_{N} \backslash\{0\}} \epsilon^{-2|p|}|p|^{2} \sigma^{2|p|}\left\{\sum_{m \equiv p}|\widehat{q}(m)|^{2} \delta^{2|m|} \underline{m}^{2 t}\right\} \\
& \times\left\{|p|^{-2(t+1)}(\sigma \delta)^{-2|p|}+C_{\sigma \delta} N^{-2(t+1)}(\sigma \delta)^{-2 N+2|p|}\right\} \\
= & C_{\epsilon, s, t} N^{2(s-t)}\left(\frac{\epsilon}{\delta}\right)^{N} \sum_{p \in \Lambda_{N} \backslash\{0\}}\left\{\sum_{m \equiv p}|\widehat{q}(m)|^{2} \delta^{2|m|} \underline{m}^{2 t}\right\} \\
& \times\left\{\left(\frac{N}{|p|}\right)^{2 t}(\delta \epsilon)^{N-2|p|}+C_{\sigma \delta}\left(\frac{|p|}{N}\right)^{2}\left(\frac{\epsilon}{\sigma^{2} \delta}\right)^{N-2|p|}\right\} \\
\leq & \left.C_{\epsilon, s, t}\|q\|_{\delta, t}^{2} N^{2(s-t)}\left(\frac{\epsilon}{\delta}\right)^{N}\right\} \\
& \times \sup _{p \in \Lambda_{N} \backslash\{0\}}\left\{\left(\frac{N}{|p|}\right)^{2 t}(\delta \epsilon)^{N-2|p|}+C_{\sigma \delta}\left(\frac{|p|}{N}\right)^{2}\left(\frac{\epsilon}{\sigma^{2} \delta}\right)^{N-2|p|}\right\} \\
\leq & C_{\epsilon, s, \delta, t, \sigma}\|q\|_{\delta, t}^{2}\left(\frac{\epsilon}{\delta}\right)^{N} \times\left\{\begin{array}{l}
N^{2 s} \quad \text { if } \delta \epsilon=1 \text { and } t>0 \\
N^{2(s-t)} \text { otherwise. }
\end{array}\right.
\end{aligned}
$$

Regarding $T_{4}$, we have

$$
\begin{aligned}
\widehat{q}(n)-\widehat{q}_{N}(n) & =\widehat{q}(n)-\frac{N}{4 \pi \varphi_{n}^{(N)}(\rho)} \sum_{m \equiv n} \frac{\sigma^{-|m|}}{|m|} \widehat{q}(m) \\
& =\frac{N}{4 \pi \varphi_{n}^{(N)}(\rho)} \sum_{\substack{m \equiv n \\
m \neq n}} \frac{\sigma^{-|m|}}{|m|}\{\widehat{q}(n)-\widehat{q}(m)\},
\end{aligned}
$$

where we used

$$
\varphi_{n}^{(N)}(\rho)=\frac{N}{4 \pi} \sum_{m \equiv n} \frac{\sigma^{-|m|}}{|m|},
$$

and

$$
T_{4} \leq \sum_{n \in \Lambda_{N} \backslash\{0\}}\left\{\frac{N}{4 \pi \varphi_{n}^{(N)}(\rho)}\right\}^{2}\left|\sum_{\substack{m \equiv n \\ m \neq n}} \frac{\sigma^{-|m|}}{|m|}\{\widehat{q}(n)-\widehat{q}(m)\}\right|^{2} \epsilon^{2|n|}|n|^{2 s}
$$




$$
\begin{aligned}
& \leq 2 \sum_{n \in \Lambda_{N} \backslash\{0\}}(\sigma \epsilon)^{2|n|}|n|^{2(s+1)}\left\{\left|\sum_{\substack{m \equiv n \\
m \neq n}} \frac{\sigma^{-|m|}}{|m|} \widehat{q}(m)\right|^{2}+\left|\sum_{\substack{m=n \\
m \neq n}} \frac{\sigma^{-|m|}}{|m|} \widehat{q}(n)\right|^{2}\right\} \\
& \equiv 2\left(T_{41}+T_{42}\right),
\end{aligned}
$$

where we used

$$
\varphi_{n}^{(N)}(\rho)=\frac{N}{4 \pi} \sum_{m \equiv n} \frac{\sigma^{-|m|}}{|m|} \geq \frac{N \sigma^{-|n|}}{4 \pi|n|}
$$

on the second inequality. $T_{41}$ is estimated as follows. We have

$$
\begin{aligned}
\left|\sum_{\substack{m \equiv n \\
m \neq n}} \frac{\sigma^{-|m|}}{|m|} \widehat{q}(m)\right|^{2} & \leq\left\{\sum_{\substack{m \equiv n \\
m \neq n}}|\widehat{q}(m)| \delta^{|m|} \underline{m}^{t} \cdot \frac{|m|^{-(t+1)}}{(2 \pi)^{t}}(\sigma \delta)^{-|m|}\right\}^{2} \\
& \leq \frac{1}{(2 \pi)^{2 t}}\left\{\sum_{\substack{m \equiv n \\
m \neq n}}|\widehat{q}(m)|^{2} \delta^{2|m|} \underline{m}^{2 t}\right\}\left\{\sum_{\substack{m \equiv n \\
m \neq n}}(\sigma \delta)^{-2|m|}|m|^{-2(t+1)}\right\}
\end{aligned} .
$$

(b)

Since the part (b) is convergent since $(\sigma \delta, t+1)>(1,1 / 2)$ and estimated as

$$
\text { (b) } \leq \sum_{\substack{l \in \mathbb{Z} \\ l \neq 0}}(\sigma \delta)^{-2|N l+n|}|N l+n|^{-2(t+1)} \leq C_{\sigma \delta} N^{-2(t+1)}(\sigma \delta)^{-2(N-|n|)} \text {, }
$$

we have

$$
\text { the right-hand side of }(50) \leq C_{\sigma \delta, t}^{\prime} N^{-2(t+1)}(\sigma \delta)^{-2(N-|n|)} \sum_{\substack{m \equiv n \\ m \neq n}}|\widehat{q}(m)|^{2} \delta^{2|m|} \underline{m}^{2 t} \text {, }
$$

and

$$
\begin{aligned}
T_{41} \leq & C_{\sigma \delta, t}^{\prime} N^{-2(t+1)}(\sigma \delta)^{-2 N} \\
& \times \sum_{n \in \Lambda_{N} \backslash\{0\}}(\sigma \delta)^{2|n|}(\sigma \epsilon)^{2|n|}|n|^{2(s+1)} \sum_{\substack{m \equiv n \\
m \neq n}}|\widehat{q}(m)|^{2} \delta^{2|m|} \underline{m}^{2 t} \\
= & C_{\sigma \delta, t}^{\prime} N^{2(s-t)}\left(\frac{\epsilon}{\delta}\right)^{N}
\end{aligned}
$$




$$
\begin{aligned}
& \times \sum_{n \in \Lambda_{N} \backslash\{0\}}\left(\frac{|n|}{N}\right)^{2(s+1)}\left(\sigma^{2} \delta \epsilon\right)^{-(N-2|n|)} \sum_{\substack{m \equiv n \\
m \neq n}}|\widehat{q}(m)|^{2} \delta^{2|m|} \underline{m}^{2 t} \\
\leq & C_{\sigma \delta, t}^{\prime} N^{2(s-t)}\left(\frac{\epsilon}{\delta}\right)^{N}\|q\|_{\delta, t}^{2} \cdot \sup _{\substack{n \in \Lambda_{N} \backslash\{0\} \\
N}}\left\{\left(\frac{|n|}{N}\right)^{2(s+1)}\left(\sigma^{2} \delta \epsilon\right)^{-(N /|n|-2)}\right\} \\
\leq & C_{\sigma \delta, t}^{\prime \prime}\|q\|_{\delta, t}^{2}\left(\frac{\epsilon}{\delta}\right)^{N} \times \begin{cases}N^{-2(t+1)} \text { if } \delta \epsilon=\sigma^{-2} \text { and } s \leq-1 \\
N^{2(s-t)} & \text { otherwise. }\end{cases}
\end{aligned}
$$

$T_{42}$ is estimated as follows.

$$
\begin{aligned}
T_{42} & =\sum_{n \in \Lambda_{N} \backslash\{0\}}(\sigma \delta)^{2|n|}|n|^{2(s+1)}\left|\sum_{\substack{m \equiv n \\
m \neq n}} \frac{\sigma^{-|m|}}{|m|}\right|^{2}|\widehat{q}(n)|^{2} \\
& \leq \sum_{n \in \Lambda_{N} \backslash\{0\}}(\sigma \epsilon)^{2|n|}|n|^{2(s+1)} \cdot C_{\sigma} N^{-2} \sigma^{-2 N+2|n|} \cdot|\widehat{q}(n)|^{2} \\
& \leq C_{\sigma, t} N^{2(s-t)}\left(\frac{\epsilon}{\delta}\right)^{N} \sum_{n \in \Lambda_{N} \backslash\{0\}}|\widehat{q}(n)|^{2} \delta^{2|n|} \underline{n}^{2 t} \cdot\left(\frac{|n|}{N}\right)^{2(s-t+1)}\left(\frac{\delta}{\sigma^{2} \epsilon}\right)^{N-2|n|} \\
& \leq C_{\sigma, t} N^{2(s-t)}\left(\frac{\epsilon}{\delta}\right)^{N} \|_{\|q\|_{\delta, t}^{2}} \cdot \sup _{n \in \Lambda_{N} \backslash\{0\}}\left\{\left(\frac{|n|}{N}\right)^{2(s-t+1)}\left(\frac{\delta}{\sigma^{2} \epsilon}\right)^{N-2|n|}\right. \\
& \leq C_{\epsilon / \delta, s, t, \sigma}\|q\|_{\delta, t}^{2}\left(\frac{\epsilon}{\delta}\right)^{N} \times\left\{\begin{array}{l}
N^{-2} \text { if } \epsilon=\sigma^{-2} \delta \text { and } s-t<-1 \\
N^{2(s-t)} \text { otherwise. }
\end{array}\right.
\end{aligned}
$$

We summarize the above results as follows.

$$
\left\|q-q_{N}\right\|_{\epsilon, s}^{2}=C\|q\|_{\delta, t}^{2}\left(\frac{\epsilon}{\delta}\right)^{N}\left(N^{2 P_{1}}+N^{2 P_{2}}+N^{2 P_{3}}+N^{2 P_{41}}+N^{2 P_{42}}\right),
$$

where

$$
\begin{aligned}
& P_{1}= \begin{cases}-(t+1) & \text { if } \delta \epsilon=\sigma^{-2} \text { and } s \leq-1 \\
s-t & \text { otherwise, }\end{cases} \\
& P_{2}=s-t, \\
& P_{3}= \begin{cases}s & \text { if } \delta \epsilon=1 \text { and } t>0 \\
s-t & \text { otherwise, }\end{cases} \\
& P_{41}= \begin{cases}-(t+1) & \text { if } \delta \epsilon=\sigma^{-2} \text { and } s \leq-1 \\
s-t & \text { otherwise, }\end{cases} \\
& P_{42}= \begin{cases}-1 & \text { if } \epsilon=\sigma^{-2} \delta \text { and } s-t<-1 \\
s-t & \text { otherwise. }\end{cases}
\end{aligned}
$$

Therefore we obtain the lemma. 
Open Access This article is distributed under the terms of the Creative Commons Attribution License which permits any use, distribution, and reproduction in any medium, provided the original author(s) and the source are credited.

\section{References}

1. Arnold, D.N.: A spline-trigonometric Galerkin method and an exponentially convergent boundary integral method. Math. Comput. 41, 383-397 (1983)

2. Henrici, P.: Applied and Computational Complex Analysis, vol. 3, Wiley-Interscience, New York (1986)

3. Katsurada, M.: Asymptotic error analysis of the charge simulation method in a Jordan region with an analytic boundary. J. Fac. Sci. Univ. Tokyo Sect. IA Math. 37, 635-657 (1990)

4. Katsurada, M.: Charge simulation method using exterior mapping functions. Japan J. Ind. Appl. Math. 11, 47-61 (1994)

5. Katsurada, M., Okamoto, H.: A mathematical study of the charge simulation method I. J. Fac. Sci. Univ. Tokyo Sect. IA Math. 35, 507-518 (1988)

6. Murota, K.: On "invariance" of schemes in the fundamental solution method. Trans. IPSJ (Information Processing Society of Japan) 34, 533-535 (1993) (in Japanese)

7. Murota, K.: Comparison of conventional and "invariant" schemes of fundamental solution method for annular domains. Japan J. Ind. Appl. Math. 12, 61-85 (1995) 